Supporting Information

\title{
Chemogenetic Evolution of a Peroxidase-like
}

\section{Artificial Metalloenzyme}

Ulrich Markel, ${ }^{\dagger,+}$ Daniel F. Sauer, ${ }^{\dagger,+}$ Malte Wittwer, ${ }^{\dagger+}$ Johannes Schiffels, ${ }^{\dagger}$ Haiyang Cui, ${ }^{\dagger}$ Mehdi D. Davari, ${ }^{\dagger}$ Konstantin W. Kröckert, ${ }^{\#}$ Sonja Herres-Pawlis, ${ }^{\#}$ Jun Okuda, ${ }^{*, \#}$ and Ulrich Schwaneberg*,,+

†Institute of Biotechnology, RWTH Aachen University, Worringerweg 3, 52074 Aachen, Germany

\#Institute of Inorganic Chemistry, RWTH Aachen University, Landoltweg 1, 52056 Aachen, Germany

${ }^{\S}$ DWI - Leibniz Institute for Interactive Materials, Forckenbeckstraße 50, 52074 Aachen, Germany These authors contributed equally.

*Corresponding authors: u.schwaneberg@biotec.rwth-aachen.de jun.okuda@ac.rwth-aachen.de

\section{Supporting Information Content}

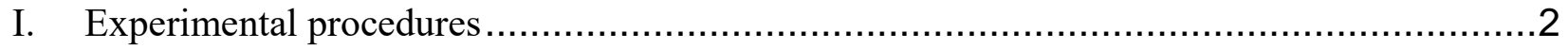

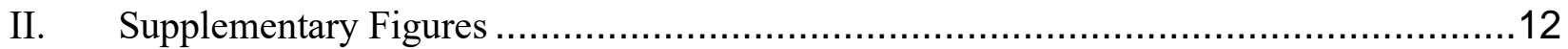

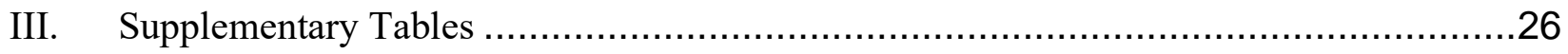

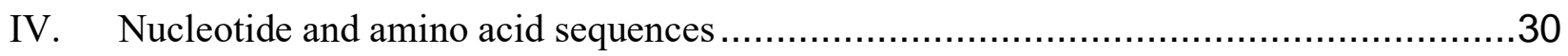

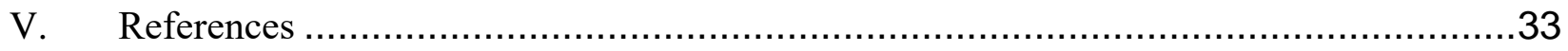




\section{Experimental procedures}

\subsection{General}

All chemicals were obtained from commercial suppliers (Sigma-Aldrich/Merck, AppliChem, and Alfa Aesar), unless otherwise noted. FePPIX (in the form of hemin) was obtained from SigmaAldrich/Merck, CoPPIX (in the form of Cobalt (III) protoporphyrin IX chloride) was obtained from Enzo Life Sciences GmbH. MnPPIX and NiPPIX were synthesized according to published procedure. ${ }^{1}$ Enzymes for molecular cloning were obtained from New England Biolabs and Thermo Fisher Scientific. Lysozyme from chicken egg white was purchased from Sigma-Aldrich.

\subsection{Computational methods}

Structural models of the MnPPIX@NB-R and MnPPIX@NB4-HR variants were constructed in YASARA Structure version $17.4 .17^{2}$ using the swap function and optimized using the SCWRL rotamer library $\operatorname{search}^{3}$ for the designed substitutions. The starting coordinate for in silico mutagenesis experiment was taken from the X-ray structure of the wildtype nitrobindin with bound heme (PDB-code: $3 \mathrm{EMM},{ }^{4}$ Resolution: $1.36 \AA$ ). The iron atom was replaced with a manganese atom. The models were further neutralized and solvated in a periodic box containing TIP3 $\mathrm{P}^{5}$ water. All energy minimizations and molecular dynamics (MD) simulations were carried out using the AMBER $14^{6}$ and GAFF force field. ${ }^{7}$ Atomic partial charges were derived using the AM1/BCC procedure $^{8}$ implemented in YASARA. Bond length to hydrogen atoms and bond angles in water were constrained to speed up the simulations. ${ }^{5}$ Electrostatics interactions were calculated using a cut-off of $7.86 \AA$ and long-range interactions were calculated by using the particle-mesh Ewald (PME) integration. Initial energy minimizations by steepest descent were followed by simulated annealing until reaching convergence in potential energy $\left(<0.02 \mathrm{~kJ} \mathrm{~mol}^{-1}\right.$ per atom during 200 steps). MD simulations were performed for $25 \mathrm{~ns}$ at $298 \mathrm{~K}$ by rescaling the time-averaged atom velocities using a Berendsen thermostat ${ }^{9}$ and a solvent density of $0.997 \mathrm{~g} \mathrm{~L}^{-1}$. During the MD simulation, coordinates and velocities were stored every 100 ps for further analysis. Root mean square fluctuation (RMSF) and distances were statistically analyzed using GROMACS analysis tools $^{10}$ after converting YASARA trajectories from .sim to .xtc. 


\subsection{Cloning and site-saturation mutagenesis}

The subcloning of NB4 was previously described by Fukumoto et al. ${ }^{11}$ Briefly, the NB4 sequence was equipped with an $N$-terminal Strep-tag II sequence and subcloned into the pET42b(+) via restriction enzymes $N d e I$ and $X h o I$. NB-wt was subcloned accordingly (all DNA and amino acid sequences are specified in chapter IV). Site-saturation mutagenesis (SSM) libraries were generated following the 22c-trick method. ${ }^{12}$ Primers were obtained from Eurofins Genomics (primer sequences are available upon request). PCR products were gel-purified (NucleoSpin Gel and PCR Clean-up kit, Macherey-Nagel), digested with DpnI, repaired using Gibson's method, ${ }^{13}$ and used to transform E. coli BL21-Gold (DE3) by electroporation. SSM libraries were extensively sequenced (Eurofins Genomics) to confirm full coverage of all 20 amino acids. In case the library did not encode for all 20 amino acids, the missing amino acid exchanges were prepared by sitedirected mutagenesis (SDM) using non-degenerated primers encoding for the missing amino acid. NB-R was prepared accordingly by SDM using non-degenerated primers and the NB-wt gene as template.

NB4-H-SpyTag was subcloned into the pET28a(+) vector using the $L g u \mathrm{I}$ restriction enzyme to assemble the NB4-H gene, the SpyTag002 sequence, and the pET28a(+) vector backbone. The NB4-H gene was equipped with terminal $L g u$ I restriction sites via PCR. An oligonucleotide consisting of the SpyTag002 and LguI recognition sites was obtained from Eurofins Genomics. Following phosphorylation and hybridization of the SpyTag002 oligonucleotide, all three fragments (vector backbone, NB4-H gene, and SpyTag002) were mixed, digested, and ligated in one pot. To introduce a single cysteine residue into the SpyCatcher, the SpyCatcher002 gene was amplified using the pMF018-Intimin-SpyCatcher002 plasmid as template and primers introducing a single cysteine codon upstream of the SpyCatcher002 gene. The PCR product was gel-purified (NucleoSpin Gel and PCR Clean-up kit, Macherey-Nagel), digested with DpnI, and then subcloned into the pET28a(+) vector using Gibson's method. ${ }^{13}$ pMF018-Intimin-SpyCatcher002 and pMF019 SpyTag002-sfGFP were kindly provided by Prof. Dr. M. Howarth and Dr. A. H. Keeble (Oxford University). 


\subsection{Protein purification}

E. coli BL21-Gold (DE3) cells freshly transformed with the gene of a nitrobindin variant (see Table S3) were grown overnight in $150 \mathrm{~mL}$ minimal medium ${ }^{14}$ supplemented with kanamycin (MM-Kan, $50 \mu \mathrm{g} / \mathrm{mL})$ at $37^{\circ} \mathrm{C}(250 \mathrm{rpm})$. The overnight culture was used to inoculate $1 \mathrm{~L}$ of MM-Kan $(50 \mu \mathrm{g} / \mathrm{mL})$. Cells were grown $\left(37^{\circ} \mathrm{C}, 200 \mathrm{rpm}\right)$ for $2.5 \mathrm{~h}$, chilled on ice for $5 \mathrm{~min}$, and gene expression was induced with Isopropyl $\beta$-D-1-thiogalactopyranoside (IPTG; final conc.: $1 \mathrm{mM}$ ). Expression was performed at $18{ }^{\circ} \mathrm{C}, 200 \mathrm{rpm}$ for $16 \mathrm{~h}$. Cells were harvested by centrifugation $\left(2,820 \mathrm{~g}, 4^{\circ} \mathrm{C}, 15 \mathrm{~min}\right)$ and cell pellets were stored at $-20^{\circ} \mathrm{C}$. Frozen cell pellets were resuspended in buffer $\mathrm{A}(50 \mathrm{mM}$ sodium phosphate $(\mathrm{NaPi}) \mathrm{pH} 8,300 \mathrm{mM} \mathrm{NaCl} ; 8 \mathrm{~mL}$ buffer per gram cell wet weight) and lysed by sonication. Crude cell lysates were centrifuged (14,000 g, $\left.4{ }^{\circ} \mathrm{C}, 30 \mathrm{~min}\right)$ and the supernatant was filtered $(0.45 \mu \mathrm{m}$ syringe filter $)$. The cleared lysate was applied twice on a gravity flow column filled with Strep-Tactin Sepharose resin (IBA Lifescience) and washed twice with 7.5 column volumes of buffer A. The target protein was eluted using buffer B (50 mM NaPi $\mathrm{pH} 8,300 \mathrm{mM} \mathrm{NaCl}, 2.5 \mathrm{mM}$ desthiobiotin). Fractions containing nitrobindin were pooled and concentrated to a total volume of $1 \mathrm{~mL}$ by ultrafiltration $(10 \mathrm{kDa}$ molecular weight cut-off, Amicon Ultra). The buffer was exchanged to reaction buffer (100 mM NaPi, pH 7.0) using a HiTrap Desalting column (GE Healcare) following the producer's manual. Purified protein was flashfrozen in liquid nitrogen and stored at $-20^{\circ} \mathrm{C}$. Nitrobindin concentration was determined by measuring the absorbance at $280 \mathrm{~nm}$ (apo-nitrobindin variants: $\varepsilon=26 \mathrm{mM}^{-1} \mathrm{~cm}^{-1}$; apo-NB4-HSpyTag: $\varepsilon=30 \mathrm{mM}^{-1} \mathrm{~cm}^{-1}$ ). An exemplary SDS-PAGE (sodium dodecyl sulfate-polyacrylamide gel electrophoresis) of all purified nitrobindin variants is shown in Supplementary Figure S17.

To produce the SpyCatcher and SpyTag-sfGFP, E. coli BL21-Gold (DE3) cells harboring the respective plasmid were grown overnight in LB medium supplemented with Kan $(50 \mu \mathrm{g} / \mathrm{mL})$ at $30{ }^{\circ} \mathrm{C}(250 \mathrm{rpm})$. The overnight culture was used to inoculate $300 \mathrm{~mL}$ of TB medium supplemented with Kan $(50 \mu \mathrm{g} / \mathrm{mL})$. Gene expression was induced at an OD of $\sim 0.8$ with IPTG $(0.2 \mathrm{mM}$ final concentration). Expression was performed at $18{ }^{\circ} \mathrm{C}, 200 \mathrm{rpm}$ for $16 \mathrm{~h}$. Cells were harvested by centrifugation $\left(2,820 \mathrm{~g}, 4{ }^{\circ} \mathrm{C}, 15 \mathrm{~min}\right)$ and cell pellets were stored at $-20{ }^{\circ} \mathrm{C}$. For purification, $1 \mathrm{~g}$ of frozen cell pellet was resuspended in $10 \mathrm{~mL}$ equilibration buffer $(20 \mathrm{mM}$ L-histidine, $0.5 \mathrm{mM}$ TCEP, pH 5.75 for the SpyCatcher, and $50 \mathrm{mM}$ Tris-HCl, $300 \mathrm{mM} \mathrm{NaCl,} \mathrm{pH} 8.0$ for SpyTagsfGFP) and lysed by sonication. The crude cell lysate was centrifuged, and the obtained cleared lysate was filtered as described above. 
The cleared lysate of SpyTag-sfGFP was loaded on a gravity-flow column filled with $\mathrm{Ni}^{2+}$-NTA resin (Qiagen). The resin was washed with 10 column volumes of equilibration buffer supplemented with $20 \mathrm{mM}$ imidazole and eluted using a concentration of $250 \mathrm{mM}$ imidazole. The buffer was exchanged to the reaction buffer, flash-frozen in liquid nitrogen, and stored at $-20{ }^{\circ} \mathrm{C}$ until further use.

The SpyCatcher was purified by ion-exchange chromatography. An ÄKTA pure 25 purification system (GE Healthcare) was equipped with a HiTrap-Q-HP column (5 mL, GE Healthcare). Following equilibration and sample application, the column was washed with 5 column volumes equilibration buffer. Proteins were eluted by a linear salt gradient (up to $1 \mathrm{M} \mathrm{NaCl}$ ). The flow rate and the gradient length was set to $3 \mathrm{~mL} / \mathrm{min}$ and $105 \mathrm{~mL}$, respectively. Eluted protein was flashfrozen in liquid nitrogen and stored at $-20^{\circ} \mathrm{C}$ until further use.

Cofactor-conjugated protein was prepared by incubating nitrobindin variants with 1.5 equiv. MnPPIX cofactor (final conc.: $88 \mu \mathrm{M}$ protein, $132 \mu \mathrm{M}$ MnPPIX, 5\% (v/v) MeCN as cosolvent) for 5 min at $23{ }^{\circ} \mathrm{C}$. Excess of cofactor was removed using a HiTrap Desalting column (GE Healcare) according to the producer's manual. Cofactor-conjugated protein was flash-frozen in liquid nitrogen and stored at $-20^{\circ} \mathrm{C}$. The concentration of MnPPIX-conjugated nitrobindin variants was determined by measuring the absorbance at $280 \mathrm{~nm}$ (MnPPIX@NB4: $\varepsilon=29,6 \mathrm{mM}^{-1} \mathrm{~cm}^{-1}$; MnPPIX@NB4-H: $\varepsilon=33$ mM cm$^{-1}$; MnPPIX@NB4-HR: $\varepsilon=32 \mathrm{mM}^{-1} \mathrm{~cm}^{-1}$; MnPPIX@NB4-HSpyTag: $\left.\varepsilon=37 \mathrm{mM}^{-1} \mathrm{~cm}^{-1}\right)$.

\subsection{ThioGlo conjugation}

To quantify the amount of NB4-H-SpyTag immobilized on the SpyCatcher-functionalized microgels, NB4-H-SpyTag was conjugated with the fluorescent dye ThioGlo. ThioGlo conjugation was performed in NaPi buffer (50 mM NaPi, $300 \mathrm{mM} \mathrm{NaCl}, 2.5 \mathrm{mM}$ d-desthiobiotin, pH 8) using $10 \mu \mathrm{M}$ NB4-H-SpyTag, 3 equiv. of ThioGlo, and 2\% (v/v) MeCN as cosolvent. After 30 min of incubation at $23{ }^{\circ} \mathrm{C}$, the sample was concentrated by ultrafiltration $(15 \mathrm{kDa}$ molecular weight cutoff, Amicon Ultra) and desalted using a PD-10 desalting column (GE Healthcare) in $100 \mathrm{mM} \mathrm{NaPi}$ buffer ( $\mathrm{pH}$ 7). The protein concentration was determined using the Pierce BCA Protein Assay (Thermo Fisher Scientific) according to the producer's manual. 


\subsection{Screening of site-saturation mutagenesis libraries}

Single colonies of E. coli BL21-Gold (DE3) transformed with a site-saturation library were used to inoculate single wells of a 96 well plate containing LB soft agar supplemented with kanamycin $(50 \mu \mathrm{g} / \mathrm{mL})$. The soft agar plate was sequenced (Eurofins Genomics) to confirm full coverage of all 20 amino acids. In case the library did not encode for all 20 amino acids, the missing amino acid exchanges were prepared by site-directed mutagenesis (SDM) using non-degenerated primers encoding for the missing amino acid. After confirming full library coverage, a glycerol stock master plate containing all possible 20 nitrobindin variants of the respective mutagenesis round was used to inoculate wells of a 96 deep-well plate containing $400 \mu \mathrm{L} /$ well of MM-Kan $(50 \mu \mathrm{g} / \mathrm{mL})$. As a control, E. coli BL21-Gold (DE3) cells transformed with pET42b(+) not containing the nitrobindin gene (“empty vector control") were used. A fresh 96 deep-well plate containing $900 \mu \mathrm{L} /$ well of MM-Kan was inoculated with $100 \mu \mathrm{L} /$ well of the preculture. Cultures were grown for $2.5 \mathrm{~h}\left(37^{\circ} \mathrm{C}\right.$, $900 \mathrm{rpm}$ ), chilled on ice for $5 \mathrm{~min}$, and supplemented with IPTG (1 mM final concentration) before further incubation $\left(18^{\circ} \mathrm{C}, 900 \mathrm{rpm}, 16 \mathrm{~h}\right)$. Cells were pelleted by centrifugation $(3,200 \mathrm{~g}, 10 \mathrm{~min}$, $4{ }^{\circ} \mathrm{C}$ ), the supernatants were discarded, and the cell pellets were stored overnight at $-20{ }^{\circ} \mathrm{C}$. The pellets were thawed for $30 \mathrm{~min}$ at room temperature, $175 \mu \mathrm{L} /$ well buffer $\mathrm{C}$ (100 mM Tris-HCl, pH 8.0, $150 \mathrm{mM} \mathrm{NaCl}$ ) supplemented with Trition X-100 (conc.: 0.5\% (v/v) were added, and the cell pellets were briefly resuspended by vortexing. $175 \mu \mathrm{L} /$ well buffer $\mathrm{C}$ (100 mM Tris-HCl, $\mathrm{pH} 8.0$, $150 \mathrm{mM} \mathrm{NaCl}$ ) containing lysozyme (final conc.: $2 \mathrm{mg} / \mathrm{mL}$ ) were added and the MTP was incubated for $1 \mathrm{~h}\left(37^{\circ} \mathrm{C}, 900 \mathrm{rpm}\right)$. Crude cell lysates were transferred to a 96 deep-well V-bottom plate and cell debris was pelleted by centrifugation $\left(3,200 \mathrm{~g}, 20 \mathrm{~min}, 4{ }^{\circ} \mathrm{C}\right)$. Quantitative SDSPAGE revealed typical nitrobindin titers of $\sim 20 \mu \mathrm{M}$ in cleared lysates (Supplementary Figure S3). $255 \mu \mathrm{L} /$ well cleared lysate were transferred to a $1.5 \mathrm{~mL}$ tube and supplemented with $30 \mu \mathrm{L}$ of MnPPIX cofactor (stock of $500 \mu \mathrm{M}$ in $\mathrm{MeOH}$; corresponds to 3 equiv. with respect to nitrobindin scaffold protein), incubated $5 \mathrm{~min}$ at $23{ }^{\circ} \mathrm{C}$, and centrifuged $(1 \mathrm{~min}, 10,000 \mathrm{~g}$ ) to remove any precipitates. Into a Chromafil-Multi 96-well filter plate (Macherey-Nagel GmbH) mounted on a vacuum manifold $50 \mu \mathrm{L} /$ well of a Strep-Tactin Sepharose suspension (50\% w/v; IBA Lifescience) were added and washed twice using $200 \mu \mathrm{L}$ buffer $\mathrm{C}$. The supernatant of the centrifuged, cofactor conjugated lysate (vide supra) was added to the Strep-Tactin Sepharose beads and the 96-well filter plate was incubated on a shaker $\left(5 \mathrm{~min}, 23{ }^{\circ} \mathrm{C}, 900 \mathrm{rpm}\right)$ before removing the liquid by vacuum 
and washing the beads twice with $200 \mu \mathrm{L}$ buffer C. Finally, the beads were resuspended in $200 \mu \mathrm{L} /$ well reaction buffer.

For the ABTS activity screening, $78 \mu \mathrm{L}$ reaction buffer, $2 \mu \mathrm{L}$ bead suspension, and $10 \mu \mathrm{L}$ ABTS (stock: $10 \mathrm{mM}$ in reaction buffer) were added to a transparent 96-well plate. Reactions were started by addition of $10 \mu \mathrm{L} \mathrm{AcOOH}$ (stock: $10 \mathrm{mM}$ ) at $23{ }^{\circ} \mathrm{C}$. The reaction progress was monitored by following the $420 \mathrm{~nm}$ absorbance signal using a Clariostar plate reader $(B M G)$ in spiral scan mode. Background activity, as determined by the empty vector control, was subtracted from library members. The libraries were screened in two independent experiments, screening each variant in duplicates, respectively.

\subsection{Comparison of metalloporphyrins in presence of different nitrobindin variants}

In a 96-well plate, $1 \mathrm{mM}$ ABTS, $25 \mathrm{nM}$ metalloporphyrin (MnPPIX, FePPIX, CoPPIX, or NiPPIX), and $50 \mathrm{nM}$ nitrobindin variant were mixed in reaction buffer containing $10 \%(\mathrm{v} / \mathrm{v}) \mathrm{MeCN}$ as cosolvent (final concentrations). Reactions were started by adding $1 \mathrm{mM}$ AcOOH or hydrogen peroxide (final concentration; total reaction volume was $100 \mu \mathrm{L} ; 23^{\circ} \mathrm{C}$ ). The reaction progress was monitored by following the absorbance at $420 \mathrm{~nm}$ using a Clariostar plate reader (BMG). As control, reactions were carried out without metalloporphyrin and the background reaction was subtracted from the other samples. The measurements were carried out in two independent experiments and each sample was measured in triplicates, respectively.

\subsection{Isothermal titration calorimetry (ITC)}

ITC was performed as described before. ${ }^{15}$ Briefly, aliquots of protein solutions (concentrations ranging from $180 \mu \mathrm{M}$ to $250 \mu \mathrm{M})$ in reaction buffer containing $1 \%(\mathrm{v} / \mathrm{v})$ DMSO as cosolvent were injected stepwise into the cell of the MicroCal PEAQ-ITC calorimeter (Malvern) containing a freshly prepared solution of the MnPPIX cofactor (concentrations ranging from $20 \mu \mathrm{M}$ to $100 \mu \mathrm{M}$ ) in reaction buffer supplemented with $1 \%(\mathrm{v} / \mathrm{v})$ DMSO as cosolvent. The binding isotherms were fitted using a one-site binding model with a fixed stoichiometry of 1 using the MicroCal PEAQITC Analysis Software (Malvern). 


\subsection{Circular dichroism spectroscopy}

Circular dichroism (CD) spectra were recorded on a JASCO J-1100 spectrometer equipped with a single position Peltier cell holder. If not stated otherwise, the temperature was set to $20^{\circ} \mathrm{C}$. The pathlength of the cuvette was $0.2 \mathrm{~mm}$. The protein concentration was $20 \mu \mathrm{M}$.

\section{$1.10 \mathrm{UV} / \mathrm{Vis}$ spectroscopy}

UV/Vis spectra were recorded on a Varian Cary 50 Bio spectrophotometer. UV-cuvettes semi micro (12.5 x 12.5 x 45 mm; polystyrene) were used (Brand, Frankfurt, Germany). To ensure equal concentrations during the measurement, $15 \mu \mathrm{M}$ MnPPIX or $15 \mu \mathrm{M}$ MnPPIX incubated with $20 \mu \mathrm{M}$ of the corresponding nitrobindin variant were used for the measurements. To measure the corresponding spectra after oxidation, the oxidant (100 equiv.) was supplemented into the cuvette placed in the machine, and the measurement was started immediately after supplementation.

Time-resolved UV/Vis spectroscopy was performed on an Avantes AvaSpec-ULS2048 CCDSpectrometer and an Avantes AvaLight-DH-S-BAL light source. The measurements were performed in Hellma QS-Screwcap-Cuvettes with an optical pathlength of $1.00 \mathrm{~cm}$. The same concentrations were used as mentioned above. The kinetic traces at $404 \mathrm{~nm}$ were used to calculate $k_{\text {obs }}$ after fitting to the following equation

$$
y=y_{0}+\left(a-y_{0}\right) \cdot\left(1-e^{-k t}\right)
$$

where $y_{0}$ is the absorbance at $t=0 \mathrm{~s}, a$ is the value of $y$ at infinite time, and $k$ is the rate constant.

\subsection{Kinetic characterization}

ABTS, syringaldehyde (SA), and 2,6-dimethoxyphenol (DMP) kinetic constants for the ArMs (MnPPIX@NB4, MnPPIX@NB4-H, and MnPPIX@NB4-HR) were determined in reaction buffer at $23{ }^{\circ} \mathrm{C}$. $K_{\mathrm{m}, \mathrm{ABTS}}$ and $k_{\mathrm{cat}, \mathrm{ABTS}}$ were determined using a concentration range of $10-600 \mu \mathrm{M}$ ABTS with a fixed concentration of $10 \mathrm{mM} \mathrm{AcOOH}$ and $25 \mathrm{nM}$ ArM. $K_{\mathrm{m}, \mathrm{SA}}$ and $k_{\mathrm{cat}, \mathrm{SA}}$ were determined using a concentration range of 10-250 $\mu \mathrm{M}$ SA with a fixed concentration of $100 \mu \mathrm{M}$ AcOOH and $0.5 \mu \mathrm{M}$ ArM. $K_{\mathrm{m}, \mathrm{DMP}}$ and $k_{\text {cat,DMP }}$ were determined using a concentration range of $10-250 \mu \mathrm{M}$ DMP with a fixed concentration of $250 \mu \mathrm{M}$ AcOOH and $0.2 \mu \mathrm{M}$ ArM. The initial reaction velocity was measured in triplicates by following the absorbance signal $\left(\varepsilon_{420}(\mathrm{ABTS})^{\circ+}=36,000 \mathrm{M}^{-1} \mathrm{~cm}^{-1}\right.$; 
$\left.\varepsilon_{469}(\mathrm{DMP})=27,500 \mathrm{M}^{-1} \mathrm{~cm}^{-1} ; \varepsilon_{364}(\mathrm{SA})=8,936 \mathrm{M}^{-1} \mathrm{~cm}^{-1}\right)$ using a Clariostar plate reader $(B M G)$. Any initial reaction velocity in the absence of ArM (background) was subtracted and the MichaelisMenten model was used for fitting employing the SciDAVis 1.22 software.

\subsection{Decolorization of recalcitrant dyes}

Decolorization experiments were performed in reaction buffer at $23{ }^{\circ} \mathrm{C}$ using $100 \mu \mathrm{M}$ dye (indigo carmine, Reactive blue 19 (RB19), or Reactive black 5 (RB5)), $100 \mu \mathrm{M}$ AcOOH and $2.5 \mu \mathrm{M}$ of MnPPIX@NB4-H or MnPPIX@NB4-HR. As control, decolorization reactions were performed without ArM. The reaction progress was monitored by following the absorbance at $610 \mathrm{~nm}$ for indigo carmine, at $595 \mathrm{~nm}$ for RB19, and at $598 \mathrm{~nm}$ for RB5 using a Clariostar plate reader (BMG). Furthermore, dye decolorizing experiments were carried out at different dye concentrations. For indigo carmine, the dye concentration was varied in the range of $40-250 \mu \mathrm{M}$ with a fixed concentration of $100 \mu \mathrm{M}$ AcOOH and $1 \mu \mathrm{M}$ ArM. For RB19, the dye concentration was varied in the range of $40-250 \mu \mathrm{M}$ with a fixed concentration of $250 \mu \mathrm{M}$ AcOOH and $0.2 \mu \mathrm{M}$ ArM. For RB5, the dye concentration was varied in the range of $10-80 \mu \mathrm{M}$ with a fixed concentration of $100 \mu \mathrm{M}$ AcOOH and $0.1 \mu \mathrm{M}$ ArM.

\subsection{Conjugation of the SpyCatcher to $N$-vinylcaprolactam-based microgels bearing epoxide moieties}

Microgels were synthesized as previously reported. ${ }^{16}$ The conjugation of the SpyCatcher to microgels was performed by adding $60 \mu \mathrm{g}$ of SpyCatcher per mg dried microgels in $\mathrm{ddH}_{2} \mathrm{O}$ containing $1 \mathrm{mM}$ Tris(2-carboxyethyl)phosphine. The mixture was placed in a shaker at $900 \mathrm{rpm}$ at $23{ }^{\circ} \mathrm{C}$ for $16 \mathrm{~h}$. The microgels-protein conjugates were centrifuged $\left(14,000 \mathrm{~g}, 18^{\circ} \mathrm{C}, 10 \mathrm{~min}\right)$, the supernatant was discarded, and the pellet was redispersed in $1 \mathrm{~mL}$ reaction buffer. Centrifugation and redispersion was repeated twice. 


\subsection{Quantification of Spy-tagged sfGFP and NB4-H-SpyTag on SpyCatcher-microgel conjugates}

A calibration curve was prepared by measuring the fluorescence intensity of different amounts of Spy-tagged sfGFP or ThioGlo-conjugated NB4-H-SpyTag in the presence of microgels. $100 \mu \mathrm{L}$ SpyCatcher-microgel conjugate ( $0.55 \mathrm{mg}$ dry microgel) were mixed with varying amounts of Spytagged sfGFP $(0-37.2 \mu \mathrm{g}=0-1.2 \mathrm{nmol})$ or ThioGlo-conjugated NB4-H-SpyTag $(0-26.1 \mu \mathrm{g}=0-1.2$ $\mathrm{nmol})$. Reaction buffer was added to a final volume of $150 \mu \mathrm{L}$. After an incubation time of $20 \mathrm{~min}$, $350 \mu \mathrm{L}$ reaction buffer were added. Following thorough mixing, $200 \mu \mathrm{L}$ of the sample were transferred to a 96-well plate and fluorescence was measured using a Clariostar plate reader $(B M G)$ (for sfGFP: $\lambda_{\mathrm{ex}}=484 \mathrm{~nm}, \lambda_{\mathrm{em}}=507 \mathrm{~nm}$; for ThioGlo-conjugated NB4-H-SpyTag: $\lambda_{\mathrm{ex}}=379 \mathrm{~nm}$, $\lambda_{\mathrm{em}}=513 \mathrm{~nm}$ ). To obtain a calibration curve, the relative fluorescence intensity was plotted against the protein concentration and linear regression was used for fitting.

For the quantification of immobilized Spy-tagged sfGFP (Figure S14a, gray squares), the same experimental setup was used but microgels were washed three times after the incubation. In the recycling experiment (Figure 4) 2.2 nmol per mg of dried MG of MnPPIX@NB4-H-SpyTag were used. To verify whether NB4-H-SpyTag is immobilized in comparable quantities to the sfGFP model system, $2.2 \mathrm{nmol}$ of a ThioGlo-NB4-H-SpyTag conjugate were applied per mg of dried MG. For washing, the microgels were centrifuged $(11,000 \mathrm{~g}, 2 \mathrm{~min})$ and redispersed in $500 \mu \mathrm{L}$ reaction buffer. The fluorescence intensity of the samples was measured as described above and the amount of immobilized protein was calculated using the respective calibration curve. The maximum protein load (Figure S14a, fit) was estimated by fitting the following five-parameter logistic (5PL) non-linear regression model to the obtained data for Spy-tagged sfGFP:

$$
y=A_{\min }+\frac{A_{\max }-A_{\min }}{\left(1+\left(\frac{x}{x o}\right)^{-h}\right)^{s}}
$$

where

$$
\begin{aligned}
& A_{\text {min }}=\text { Minimum asymptote }, \\
& A_{\text {max }}=\text { Maximum asymtptote, } \\
& x o=\text { Inflection point },
\end{aligned}
$$


$h=$ Hill'slope,

$s=$ Asymmetry factor

To quantify the amount of protein unspecifically bound to the microgel, the experiment was repeated using microgels without SpyCatcher. Unspecific absorption of the protein to the microgel was not observed (Figure S14, white squares).

\subsection{Recycling of MnPPIX@NB4-H for dye decolorization}

For the recycling experiment, $900 \mu \mathrm{L}$ SpyCatcher-microgel conjugates $(6.125 \mathrm{mg}$ dry microgel) were mixed with $290 \mu \mathrm{g}$ MnPPIX@NB4-H-SpyTag (= $2.2 \mathrm{nmol}$ protein $/ \mathrm{mg}$ of dried microgel). For a control, reaction buffer was added instead of MnPPIX@NB4-H-SpyTag. After an incubation time of $20 \mathrm{~min}$, microgels were washed three times. To this end, microgels were centrifuged $(11,000 \mathrm{~g}, 2 \mathrm{~min})$ and redispersed in $1 \mathrm{~mL}$ reaction buffer. After the final centrifugation step, microgels were redispersed in $900 \mu \mathrm{L}$ reaction buffer. Afterward, $50 \mu \mathrm{L}$ indigo carmine $(0.5 \mathrm{mM}$ stock solution) and $50 \mu \mathrm{L} \mathrm{AcOOH}$ were added ( $0.5 \mathrm{mM}$ stock solution). After 2 min of incubation, the suspension was centrifuged $(13,000 \mathrm{~g}, 1 \mathrm{~min}) .200 \mu \mathrm{L}$ of the supernatant were transferred to a 96-well plate and the absorbance of indigo carmine was measured at $610 \mathrm{~nm}$ using a Clariostar plate reader $(B M G)$. The remaining supernatant was discarded. The microgels were resuspended in $900 \mu \mathrm{L}$ of fresh reaction buffer and the reaction was started again as described above. 


\section{Supplementary Figures}

a

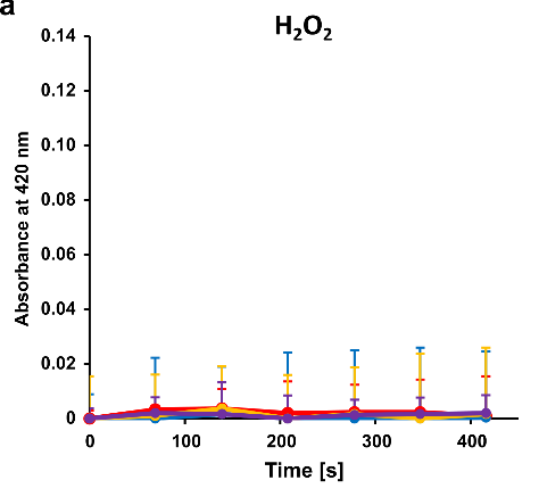

C

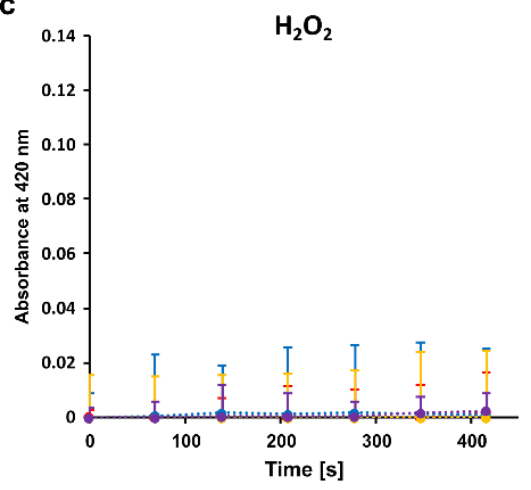

-MnPPIX@NB4

$\rightarrow$ FePPIX@NB4

$\Longrightarrow$ COPPIX@NB4

$\rightarrow$ NiPPIX@NB4

-.-.-MnPPIX@NB4-HR

..- FePPIX@NB4-HR

-. - CoPPIX@NB4-HR

..-. NiPPIX@NB4-HR b

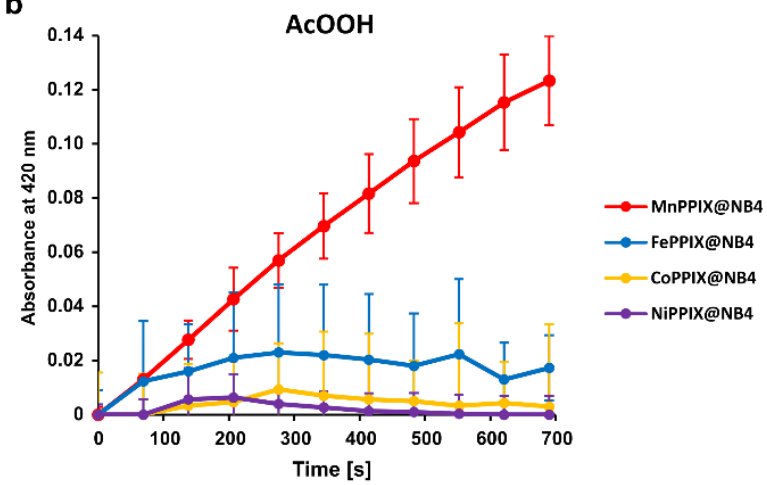

d

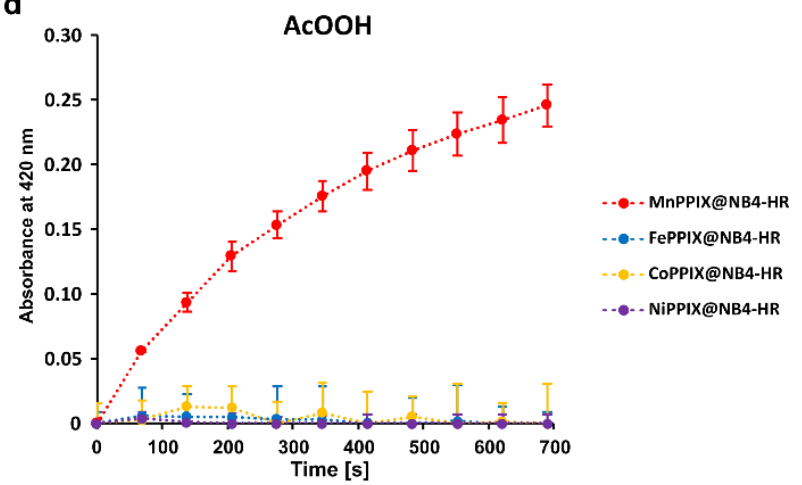

e

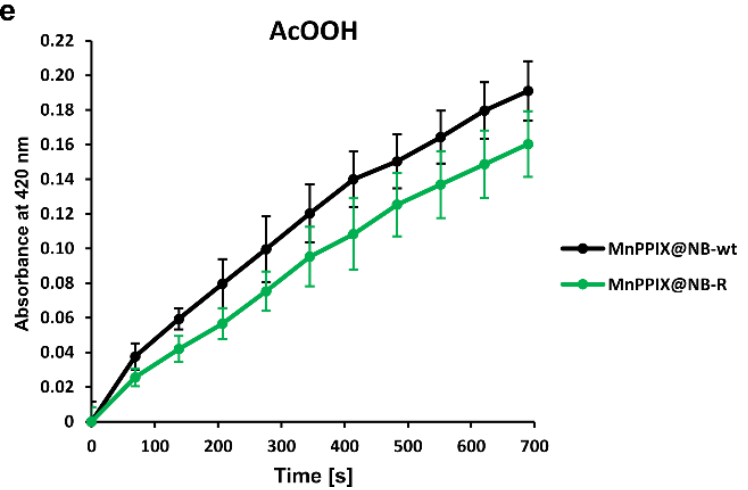

Figure S1. Time courses of ABTS oxidation. a) and b) Comparison of different metalloporphyrins (MnPPIX, FePPIX, CoPPIX, and NiPPIX) in presence of 2 equiv. of the NB4 scaffold using either hydrogen peroxide $\left(\mathrm{H}_{2} \mathrm{O}_{2}\right)$ or peracetic acid $(\mathrm{AcOOH})$ as oxidant. c) and d) Comparison of different metalloporphyrins in presence of 2 equiv. of the NB4-HR scaffold using either hydrogen peroxide $\left(\mathrm{H}_{2} \mathrm{O}_{2}\right)$ or peracetic acid (AcOOH) as oxidant. e) Comparison of MnPPIX in presence of 2 equiv. of wildtype nitrobindin (MnPPIX@NB-wt) and the wildtypederived nitrobindin variant carrying the Arg76 substitution (MnPPIX@NB-R). Experimental details are given in chapter 1.7. 
NB4

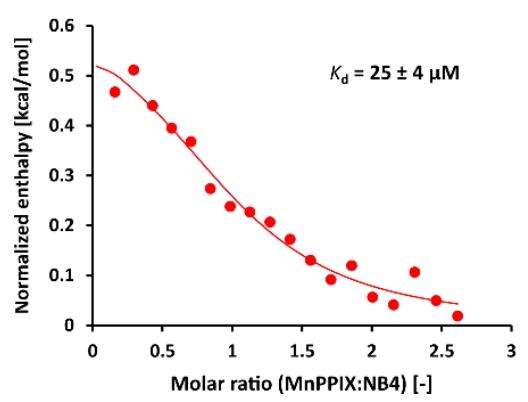

NB4-H

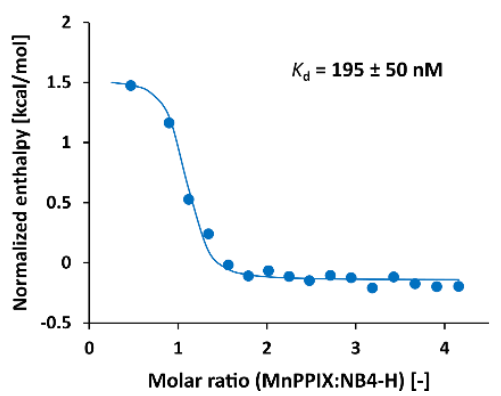

NB4-HR

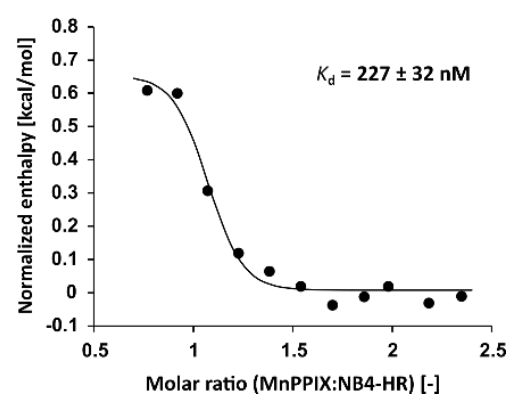

Figure S2. Isothermal titration calorimetry of MnPPIX cofactor and nitrobindin scaffold proteins (NB4, NB4-H, NB4-HR). Experimental details are given in chapter 1.8.
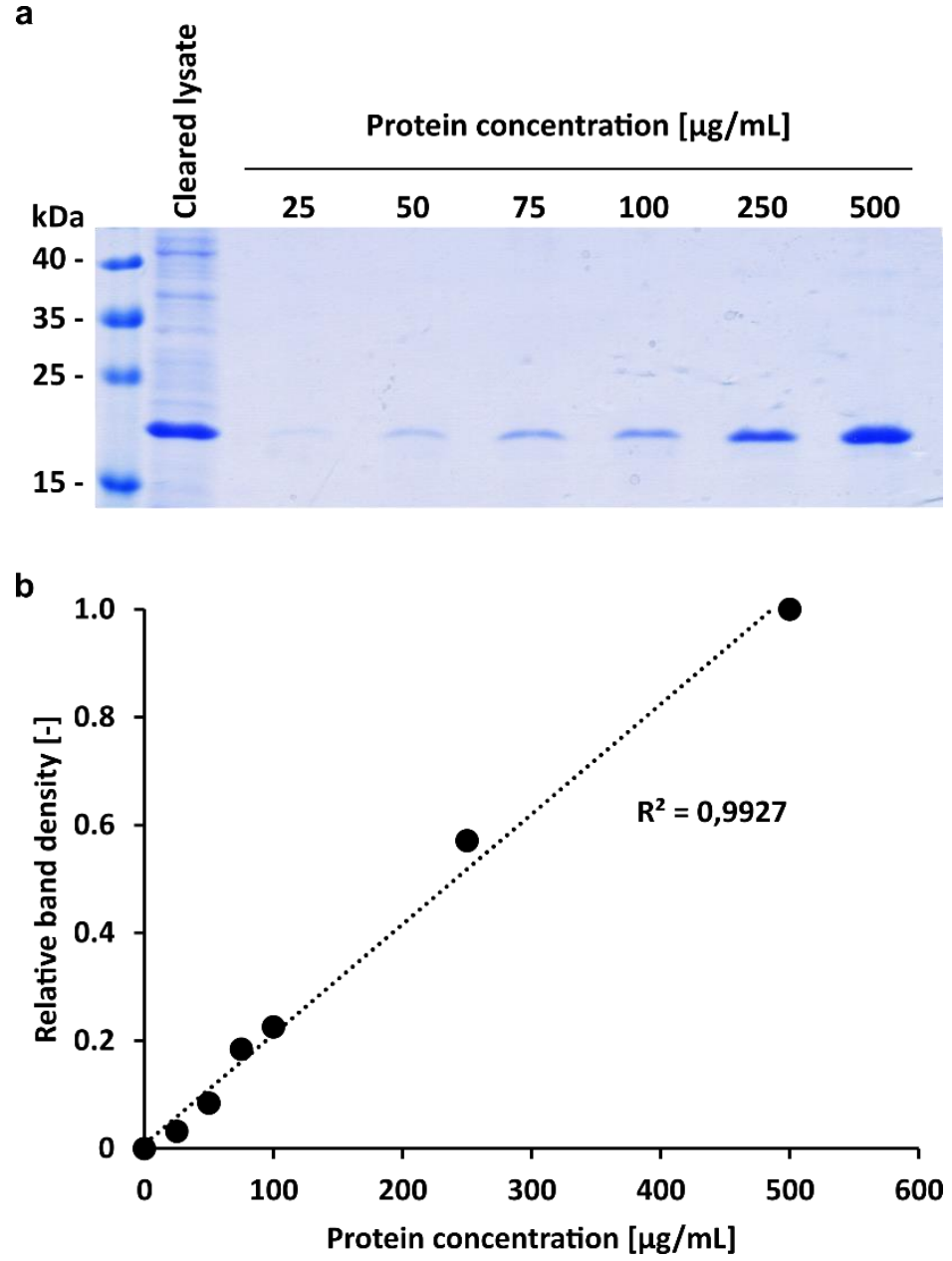

Figure S3. Densitometry-based quantification of NB4 in cleared lysates. a) Representative SDS-PAGE analysis of a sample of cleared lysate containing overexpressed NB4 $(\sim 19.5 \mathrm{kDa})$ and samples of purified NB4 at different concentrations. b) Densitometric quantification was carried out using ImageJ (version 1.52a) and revealed typical scaffold protein titers of $\sim 20 \mu \mathrm{M}$ in cleared lysates. No difference was observed for the expression levels of different NB4 variants. 

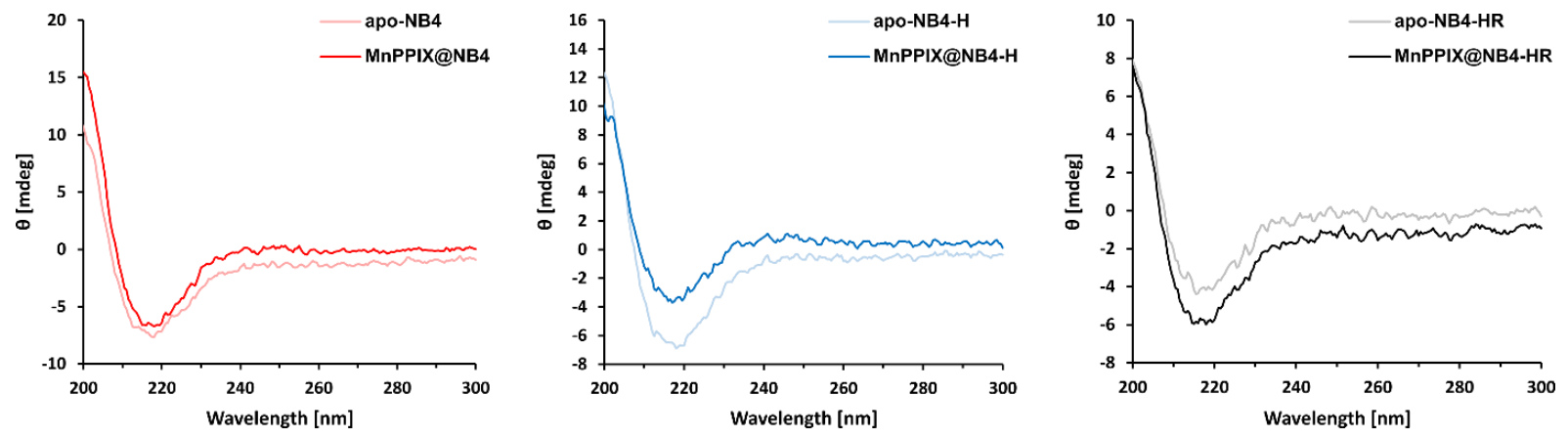

Figure S4. CD spectra of apo- and MnPPIX-conjugated form of NB4, NB4-H, and NB4-HR. The CD traces indicate structural integrity of all apo- and MnPPIX-bound nitrobindin variants. 

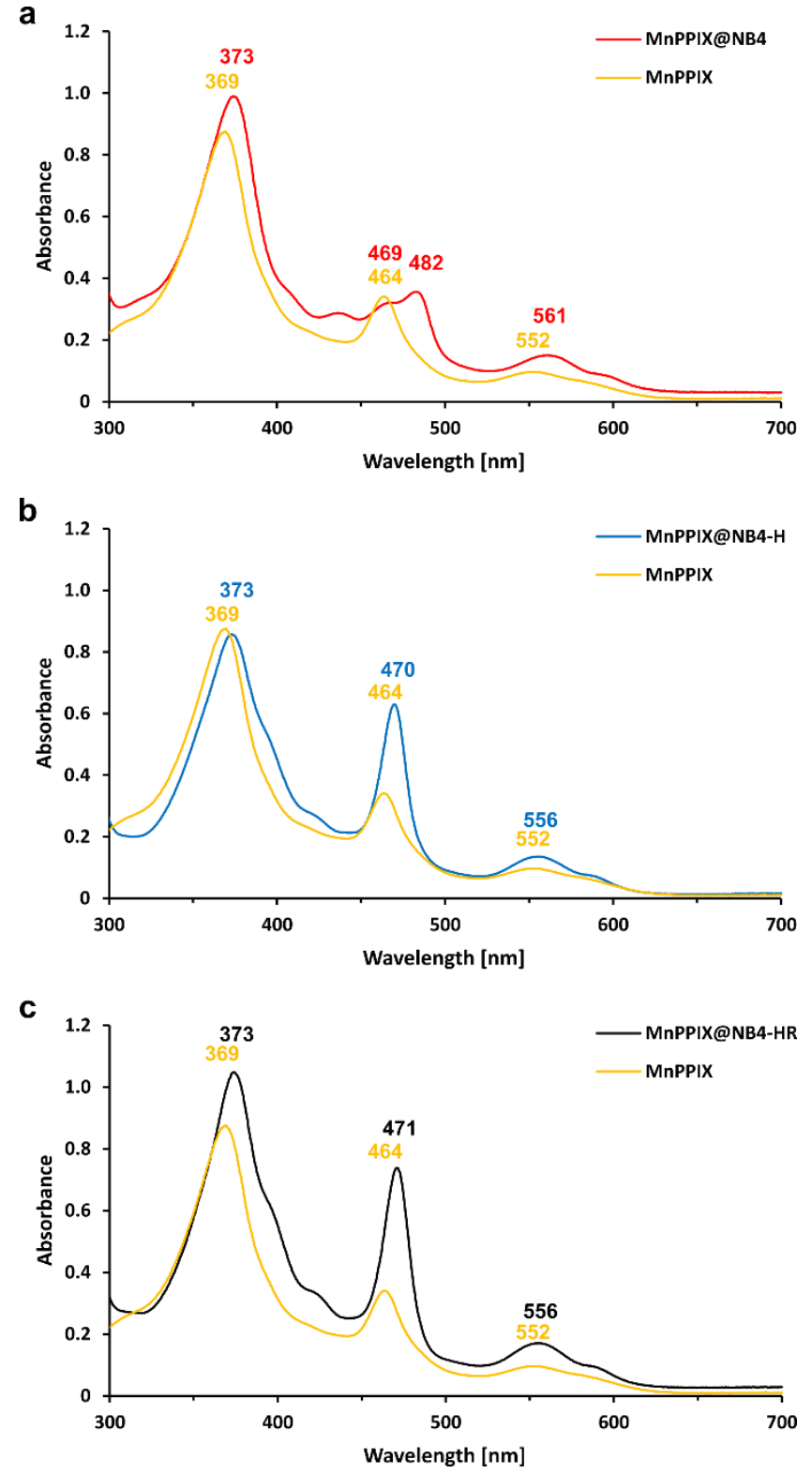

Figure S5. UV/Vis spectra of protein-free MnPPIX and MnPPIX-conjugated to NB4 (a), NB4-H (b), and NB4-HR (c). 

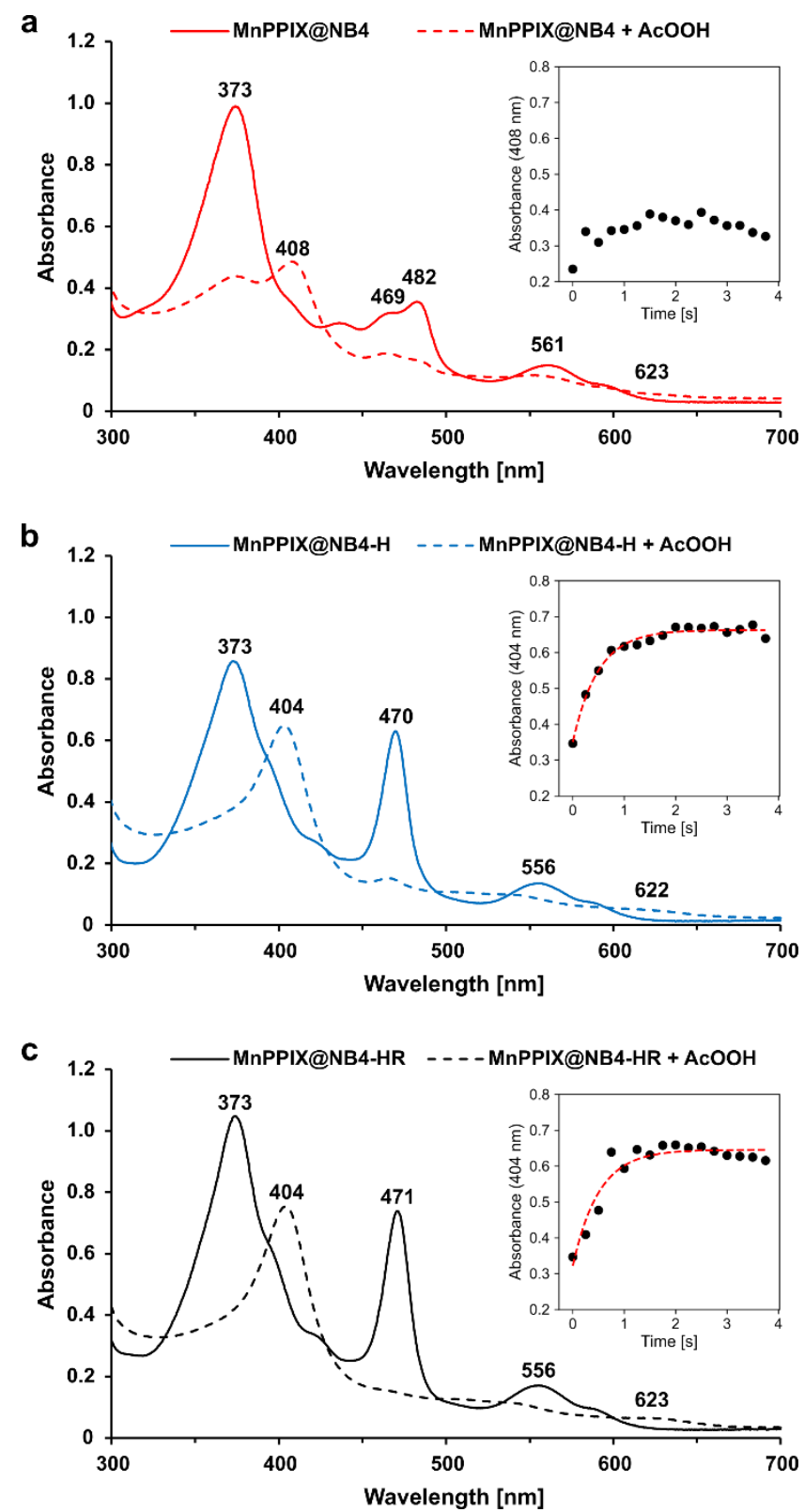

Figure S6. UV/Vis spectra of MnPPIX@NB4 (a), MnPPIX@NB4-H (b), and MnPPIX@NB4-HR (c) without $\mathrm{AcOOH}$ (solid lines) and upon addition of 100 equiv. of $\mathrm{AcOOH}$ (dashed lines). Insets: Time-resolved UV/Vis spectroscopy for the formation of $\mathrm{Mn}(\mathrm{V})=\mathrm{O} . k_{\mathrm{obs}}(\mathrm{MnPPIX} @ \mathrm{NB} 4-\mathrm{HR})=2.1 \mathrm{~s}^{-1} ; k_{\mathrm{obs}}(\mathrm{MnPPIX} @ \mathrm{NB} 4-\mathrm{H})=2.2 \mathrm{~s}^{-1}$; for MnPPIX@NB4 the time-resolved formation of $\mathrm{Mn}(\mathrm{V})=\mathrm{O}$ species was not detected. 


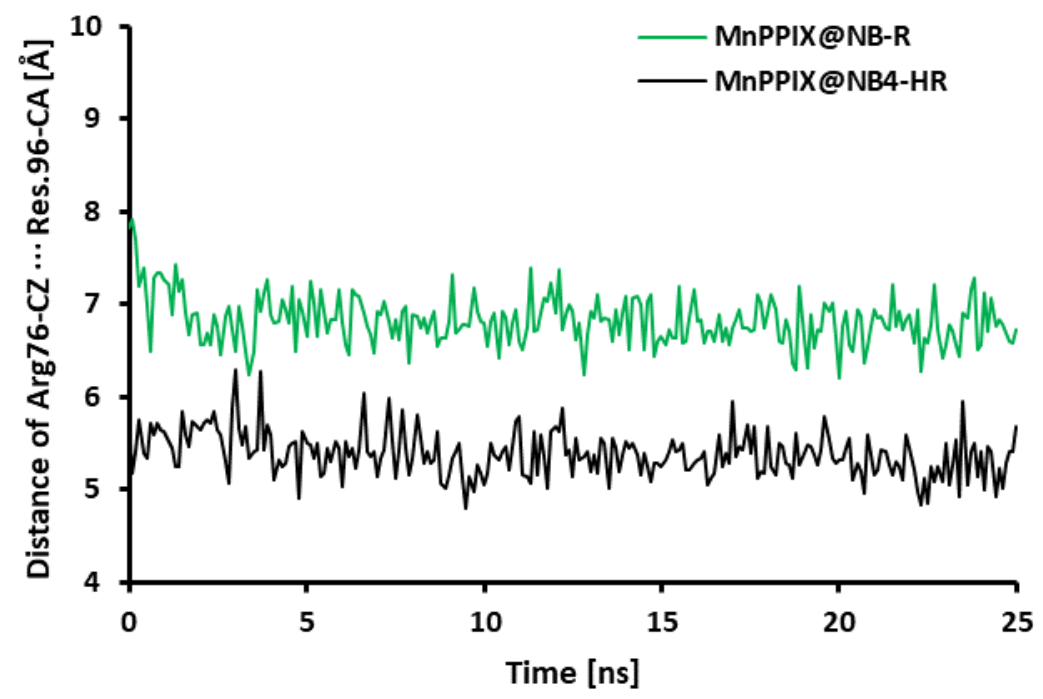

Figure S7. Analysis of the distance between Arg76 and the residue at position 96 (Gln96 in NB-R; Cys96 in NB4HR). Distances were measured from the carbon of the Arg76 guanidinium group ("Arg76-CZ") to the C $\alpha$ of the residue at position 96 ("Res.96-CA") over the course of the MD simulations. Average distances of the variants were $\sim 6.8 \AA$ for MnPPIX@NB-R, and 5.3 $\AA$ for MnPPIX@NB4-HR. The interaction with Gln96 in MnPPIX@NB-R keeps Arg76 at a larger distance from the C $\alpha$ of Gln96, while in MnPPIX@NB4-HR the sidechain at position 96 is shorter (Cys) and thus sterically less demanding. Hence, Arg76 can penetrate this free space and accommodate a more "relaxed"/“straight chain conformation" (compare Figure 3). 


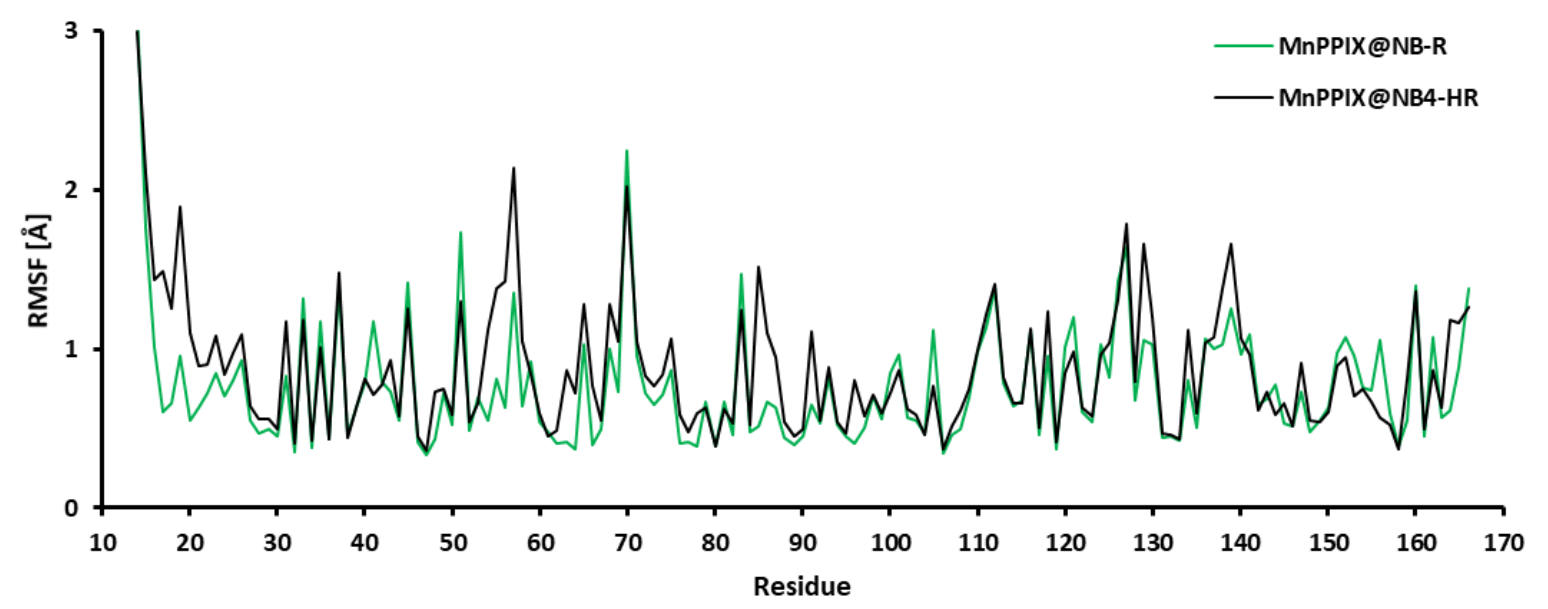

b

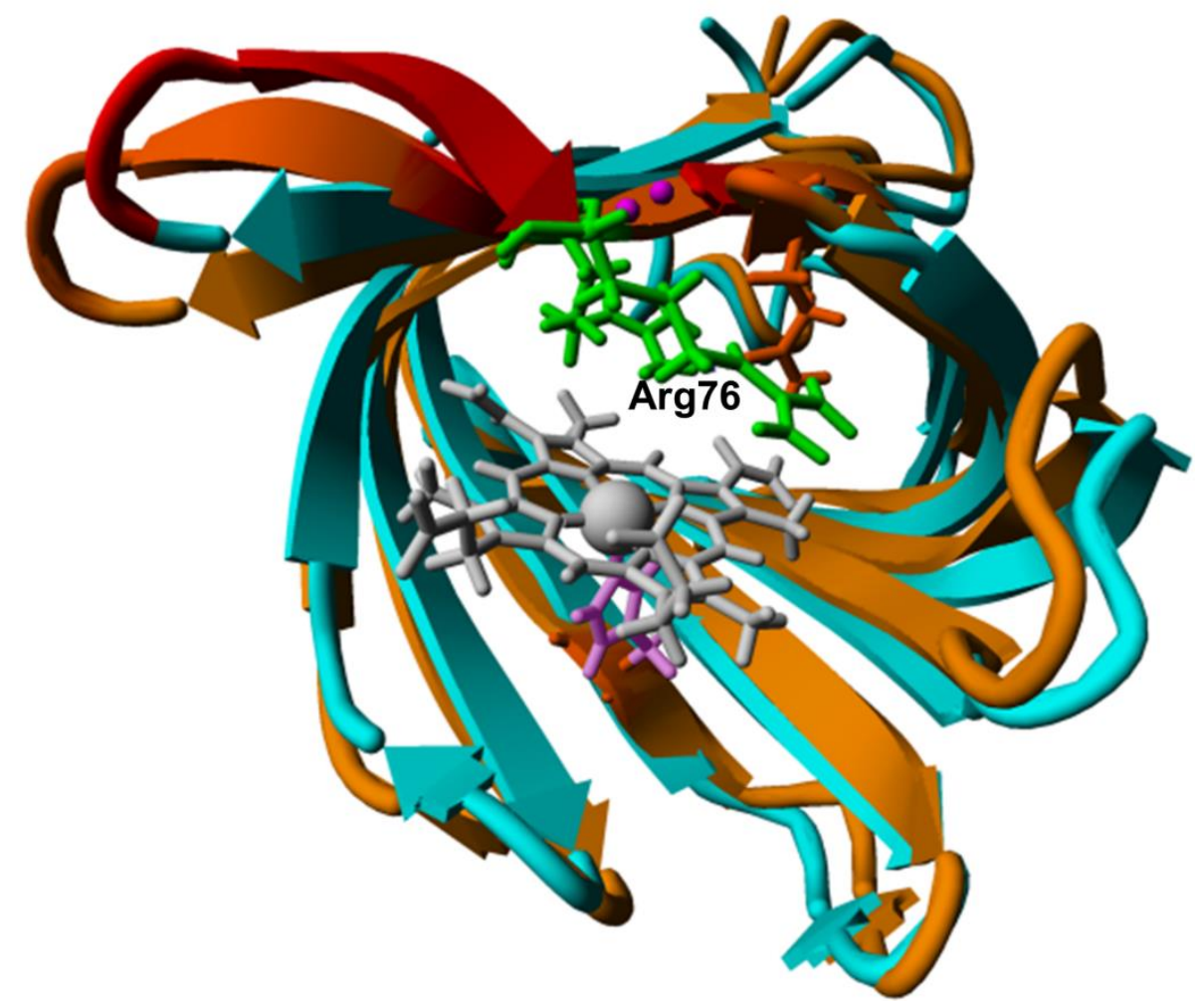

Figure S8. a) Root mean square fluctuation (RMSF) of the protein backbone of MnPPIX@NB4-HR and MnPPIX@NB-R for the last10 ns of the MD simulations. b) Enlarged front view around the MnPPIX cofactor (distal site) in the structures of MnPPIX@NB4-HR and MnPPIX@NB-R. Arg76 is colored in green sticks, His158 is colored in magenta sticks. The $\beta$-barrel structures of the variants are superimposable with a RMSD value of less than $1.0 \AA$. The $\beta$-strand harboring the Arg76 substitution in MnPPIX@NB4-HR variant is shown in red. 


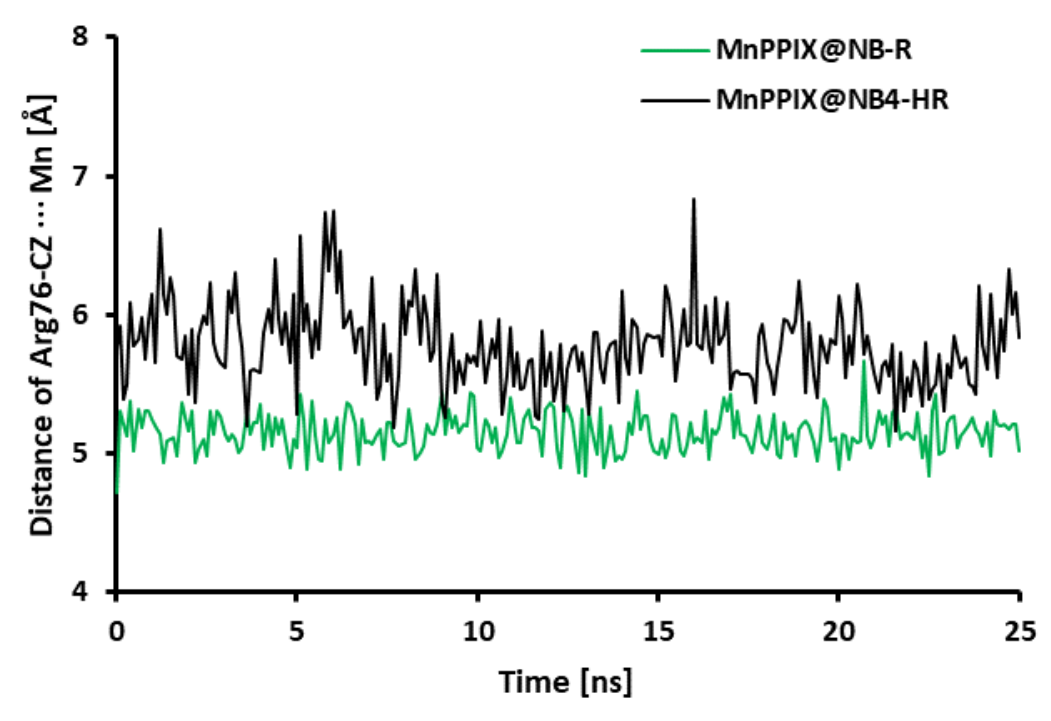

Figure S9. Analysis of the distance between Arg76 and the Mn center. Distances were measured from the carbon of the Arg76 guanidinium group ("Arg76-CZ") to the Mn center over the course of the MD simulations. Average distances of the variants were 5.1 $\AA$ for MnPPIX@NB-R and 5.8 $\AA$ for MnPPIX@NB4-HR. The distances fluctuate around the average distance by $\pm 0.3 \AA$ and $\pm 0.7 \AA$, respectively, indicating a more dynamic relationship between Arg76 and the MnPPIX cofactor in MnPPIX@NB4-HR compared to MnPPIX@NB-R. 


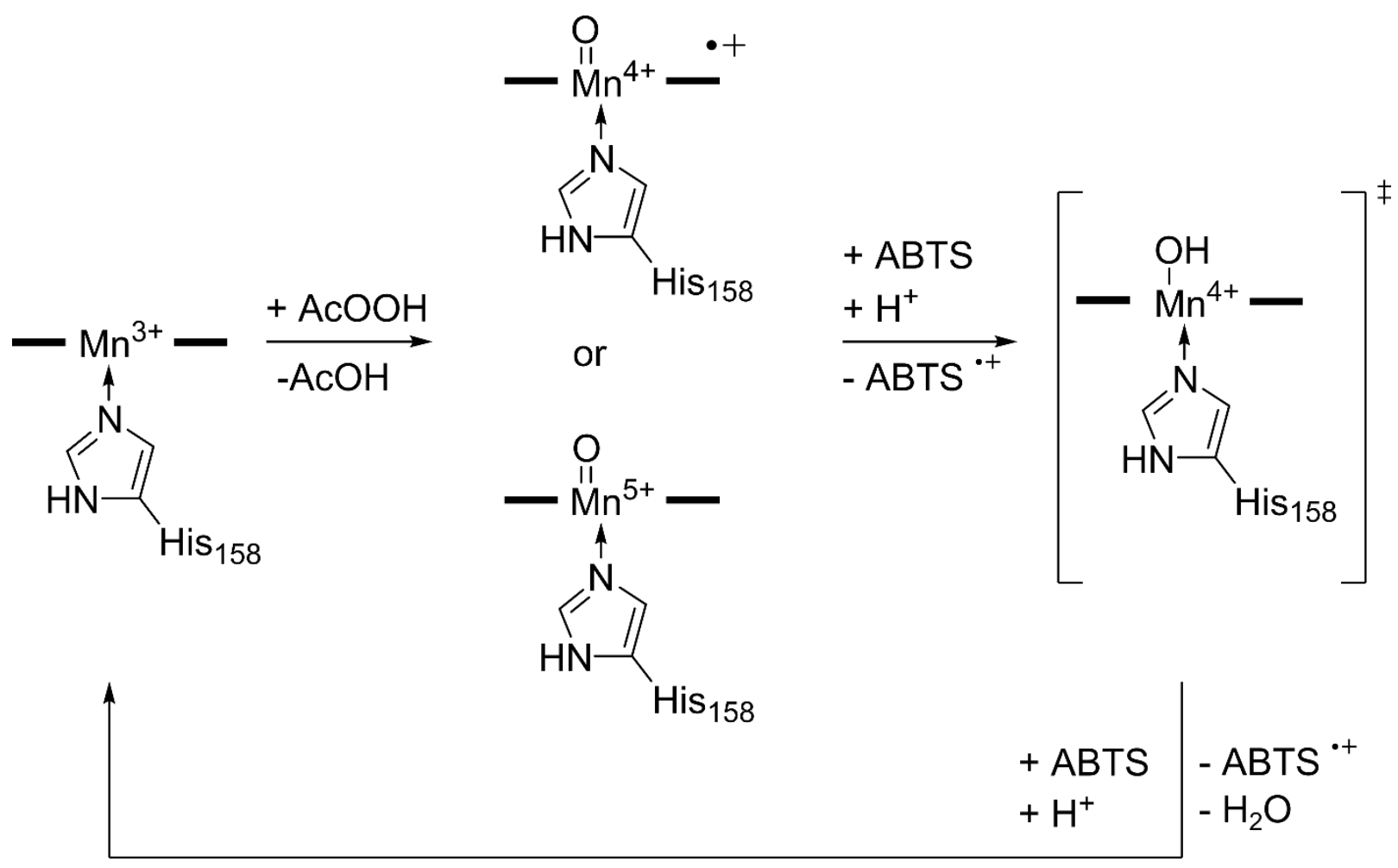

Figure S10. Proposed reaction mechanism for the oxidation of ABTS. Mn(III) in MnPPIX@NB4-HR is oxidized by $\mathrm{AcOOH}$ to formally $\mathrm{Mn}(\mathrm{V})=\mathrm{O}$ (either $\mathrm{Mn}(\mathrm{V})=\mathrm{O}$ or $[\mathrm{Mn}(\mathrm{IV})=\mathrm{O}]^{+*}$ ). ABTS is oxidized, leading to the formation of an Mn(IV)-OH species. This species is reduced once more by ABTS leading to the Mn(III)PPIX@NB4-HR complex and water, which completes the catalytic cycle.

a

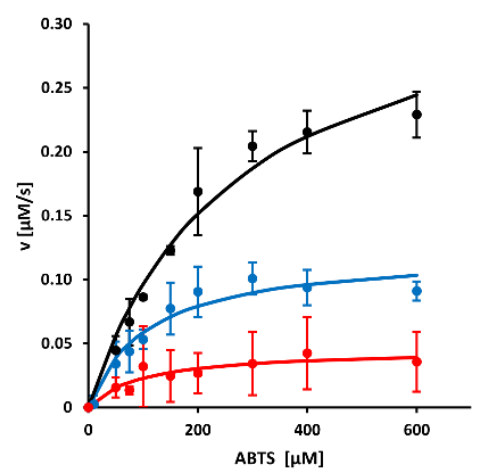

b

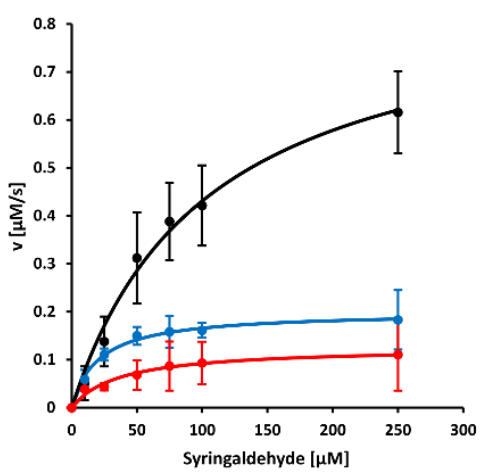

C

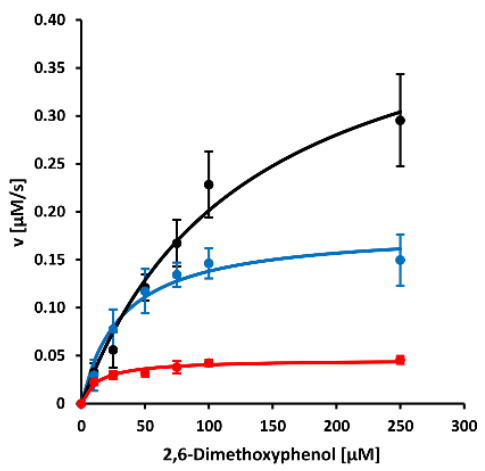

- MnPPIX@NB4

- MnPPIX@NB4-H

- MnPPIX@NB4-HR

Figure S11. Kinetic characterization of the peroxidase-like ArMs for the substrates ABTS (a), syringaldehyde (b), and 2,6-dimethoxyphenol (c) using AcOOH as oxidant. Initial reaction velocities $(v)$ were determined in triplicates. Experimental details are given in chapter 1.11. 

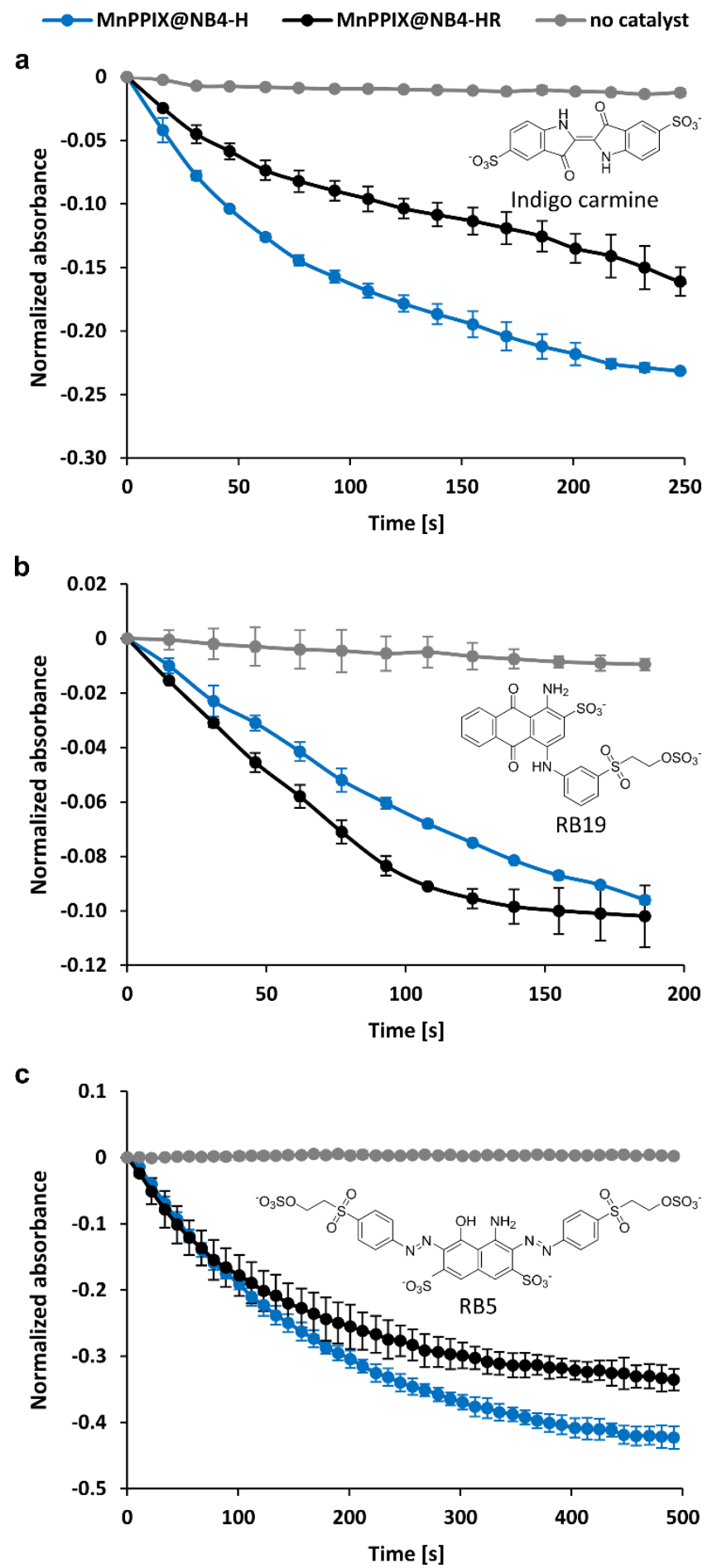

Figure S12. Dye decolorization activity of the peroxidase-like ArMs. Experiments were performed in triplicate using $100 \mu \mathrm{M}$ dye (indigo carmine (a), RB19 (b), RB5 (c)), 1 equiv. AcOOH, and $2.5 \mu \mathrm{M}$ ArM. Experimental details are given in chapter 1.12 . 
a (Indigo carmine)
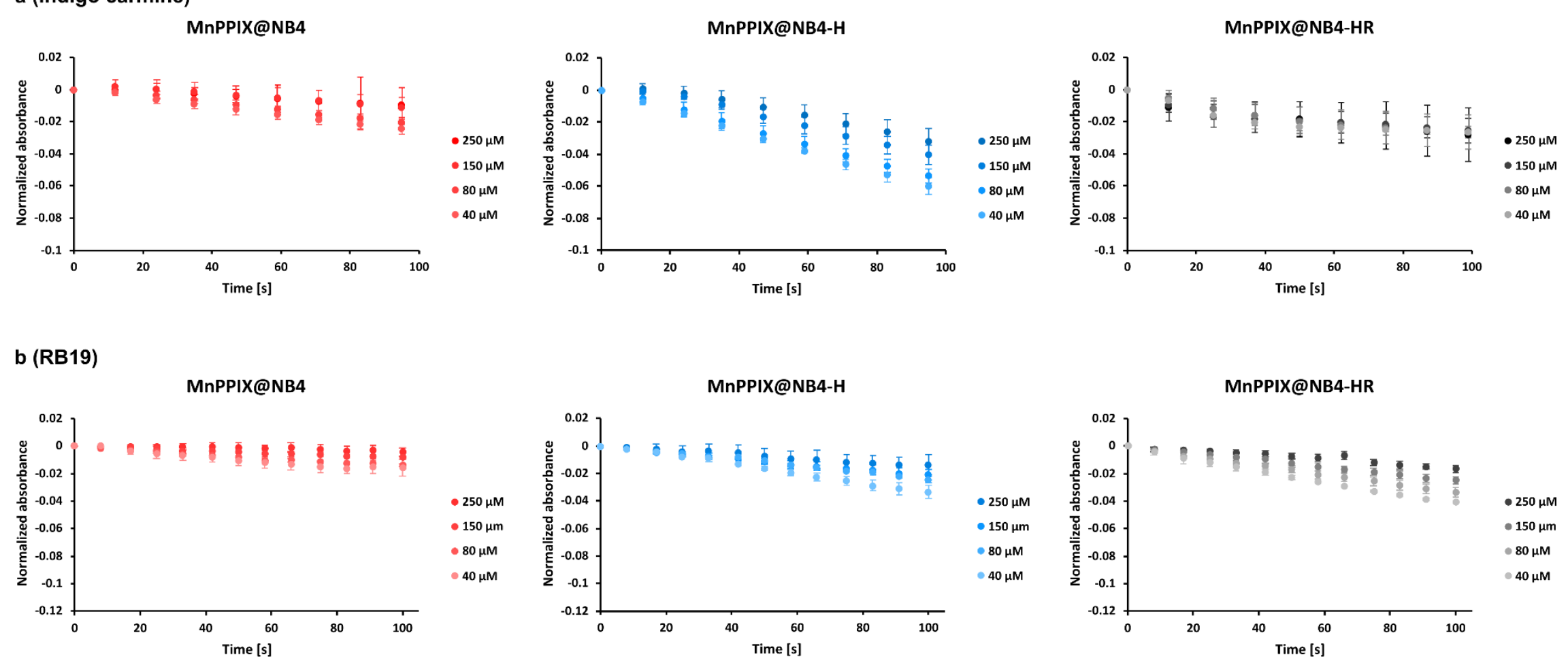

\section{c (RB5)}
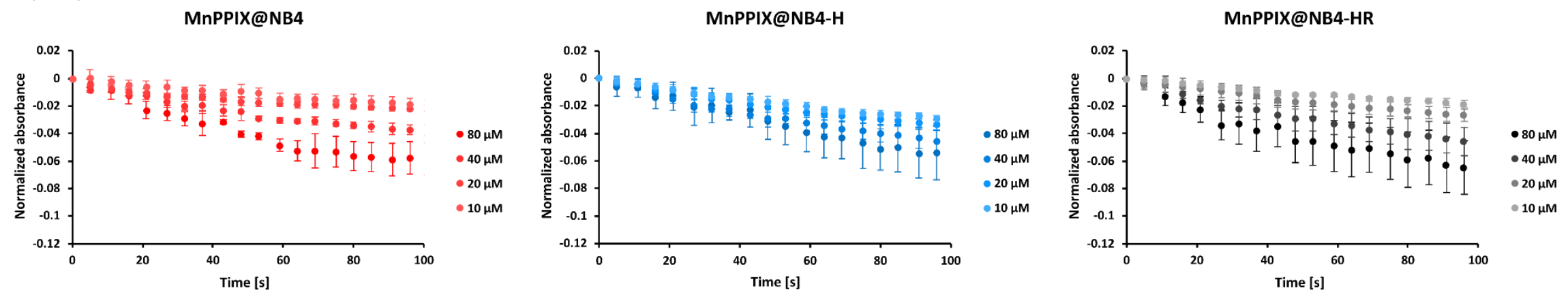

Figure S13. Dye decolorization activity at different dye concentrations. Indigo carmine (a), RB19 (b), RB5 (c) Experimental details are given in chapter 1.12. 
a

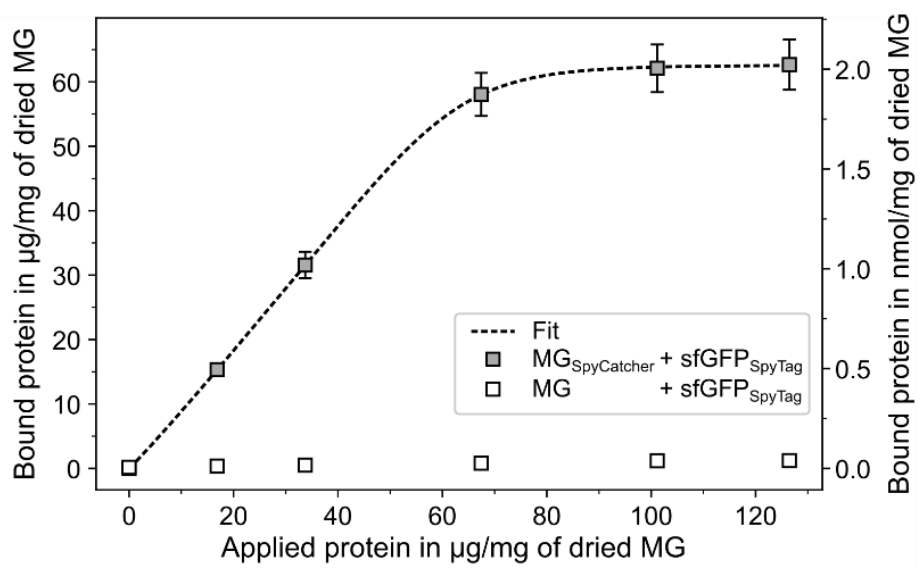

b

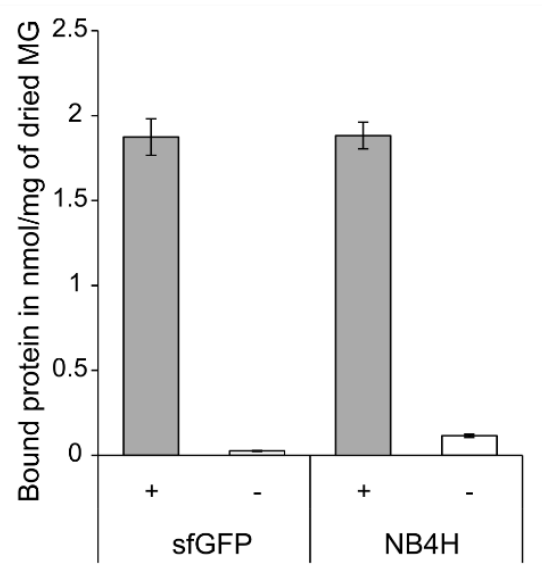

Figure S14. a) Conjugation of Spy-tagged sfGFP to SpyCatcher-functionalized microgel (MG). Being a $\beta$-barrel protein of comparable size to nitrobindin, sfGFP provides a valuable model protein for an easy fluorescent read-out of the protein immobilization. Spy-tagged sfGFP, previously shown to be capable of forming a covalent bond with SpyCatcher, ${ }^{17}$ was conjugated to the SpyCatcher-functionalized microgels. A saturation behavior was observed when the applied sfGFP concentrations exceeded $\sim 70 \mu \mathrm{g}(\sim 2.2 \mathrm{nmol})$ of sfGFP per mg of dry microgel (grey squares). At this concentration, up to $1.8 \mathrm{nmol}$ of sfGFP per $\mathrm{mg}$ of dried MG (=84\%) were successfully immobilized, which is close to the calculated maximum amount of immobilized sfGFP per mg of dried microgel $(2.0 \mathrm{nmol})$. No adsorption of the protein to the microgel was observed when microgels without SpyCatcher were used (white squares). Thus, in the recycling experiment (Figure 4) 2.2 nmol per mg of dried MG of MnPPIX@NB4H-SpyTag were used. b) To verify that NB4-H-SpyTag is immobilized in comparable quantities to the sfGFP model system, $2.2 \mathrm{nmol}$ of a ThioGlo-NB4-H-SpyTag conjugate were applied per $\mathrm{mg}$ of dried MG. "+" indicates the use of MG functionalized with SpyCatcher and "_. indicates the use of MG that were not functionalized with SpyCatcher. Results of the corresponding sfGFP data points are shown for comparison. Indeed, a comparable amount (1.9 nmol per mg of dried MG) of ThioGlo-NB4-H-SpyTag was immobilized. Also, the ThioGlo-NB4-HSpyTag conjugate showed only low unspecific binding to the MG. 


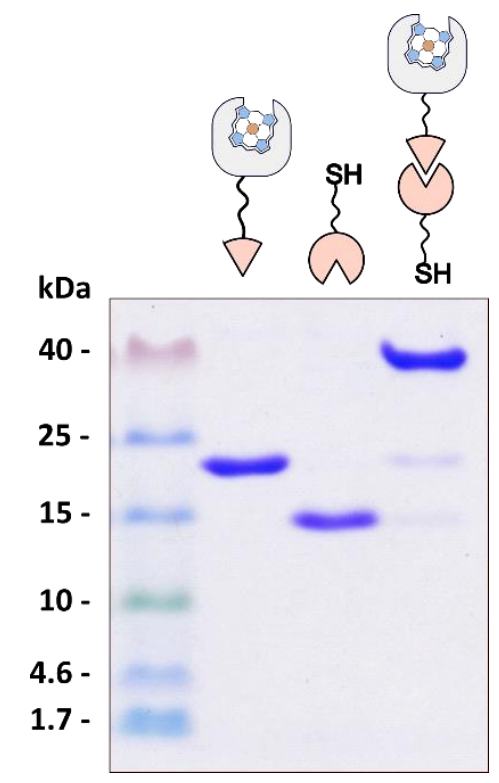

Figure S15. SDS-PAGE analysis of the SpyTag-SpyCatcher conjugation. First lane: MnPPIX@NB4-H-SpyTag $(22 \mathrm{kDa})$. Second lane: SpyCatcher $(14 \mathrm{kDa}$; “-SH" indicates the single cysteine residue present in the SpyCatcher variant used in this study, which was designated for the conjugation of the SpyCatcher to the microgel). Third lane: Equimolar amounts of MnPPIX@NB4-H-SpyTag and SpyCatcher were mixed. The appearance of a new band (lane three) indicates the successful formation of the covalent bond between the SpyTag and SpyCatcher moieties ultimately leading to the conjugation of MnPPIX@NB4-H-SpyTag and SpyCatcher (conjugate: $36 \mathrm{kDa}$ ).

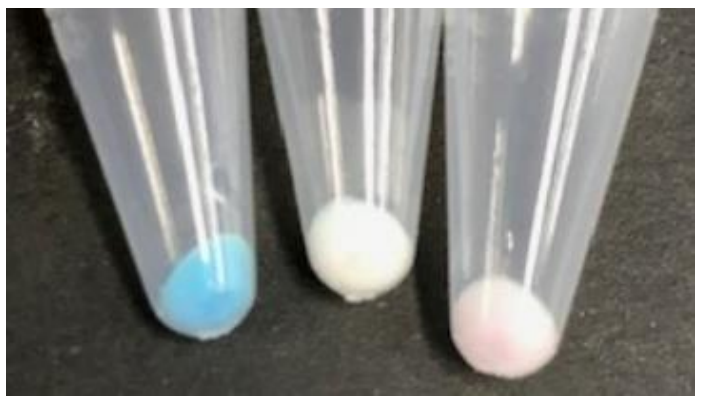

Figure S16. Pellets of ArM-microgel conjugates. Left: SpyCatcher-functionalized microgels without ArM after one round of indigo carmine decolorization. The blue indigo carmine dye accumulates in the microgels, leading to an apparent decolorization of the supernatant; middle: microgels before the addition of indigo carmine; right: ArMfunctionalized microgels after one round of indigo carmine decolorization. Here, the indigo carmine dye is decolorized by the ArM. 


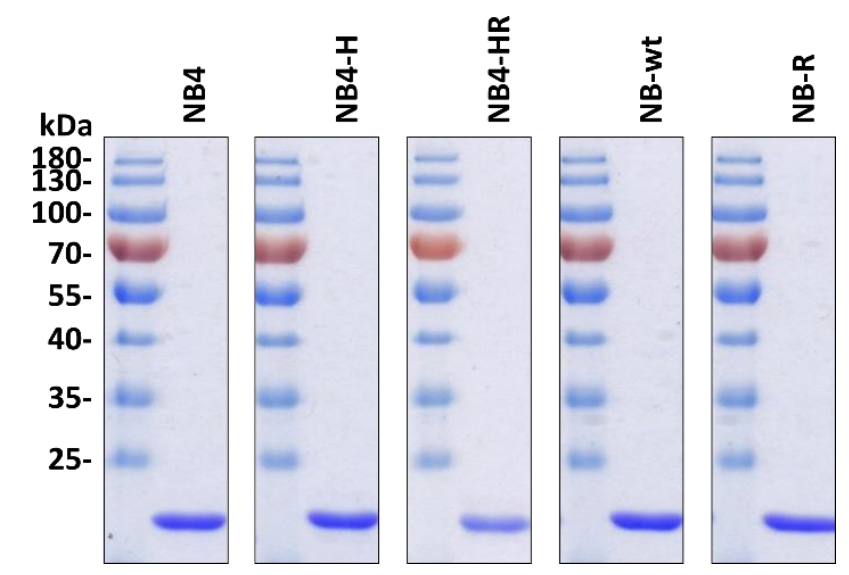

Figure S17. SDS-PAGE of purified nitrobindin variants. 


\section{Supplementary Tables}

Table S1. Screening results of the first round of directed evolution (SSM 158 on NB4).

\begin{tabular}{|c|c|c|}
\hline Amino acid substitution & Initial rea & action rate $[\mathrm{mAU} / \mathrm{s}]^{\mathrm{a})}$ \\
\hline $\mathrm{F}$ & 1.02 & \pm 0.02 \\
\hline $\mathrm{Y}$ & 1.20 & \pm 0.10 \\
\hline $\mathrm{W}$ & 1.31 & \pm 0.21 \\
\hline A & 1.07 & \pm 0.05 \\
\hline V & 1.19 & \pm 0.01 \\
\hline $\mathrm{L}$ & 0.99 & \pm 0.12 \\
\hline I & 1.29 & \pm 0.09 \\
\hline G & 1.14 & \pm 0.11 \\
\hline $\mathrm{C}$ & 1.42 & \pm 0.02 \\
\hline M & 1.33 & \pm 0.04 \\
\hline $\mathrm{P}$ & 1.17 & \pm 0.02 \\
\hline $\mathrm{S}$ & 1.65 & \pm 0.02 \\
\hline $\mathrm{T}$ & 1.77 & \pm 0.05 \\
\hline $\mathrm{N}$ & 1.83 & \pm 0.16 \\
\hline Q & 1.77 & \pm 0.06 \\
\hline $\mathrm{D}$ & 1.20 & \pm 0.05 \\
\hline $\mathrm{E}$ & 1.18 & \pm 0.09 \\
\hline $\mathrm{H}$ & 2.21 & \pm 0.00 \\
\hline K & 1.59 & \pm 0.18 \\
\hline $\mathrm{R}$ & 1.81 & \pm 0.10 \\
\hline EV & 0.70 & \pm 0.02 \\
\hline
\end{tabular}


Table S2. Screening results of the second round of directed evolution (SSM 76 on NB4-H).

\begin{tabular}{rrr}
\hline Amino acid substitution & Initial reaction rate $[\mathbf{m A U} / \mathbf{s}]^{\mathbf{a})}$ \\
\hline F & 1.23 & \pm 0.10 \\
Y & 2.09 & \pm 0.41 \\
W & 1.53 & \pm 0.06 \\
A & 1.45 & \pm 0.24 \\
V & 2.39 & \pm 0.33 \\
L & 1.39 & \pm 0.13 \\
I & 1.67 & \pm 0.33 \\
G & 1.78 & \pm 0.12 \\
C & 1.69 & \pm 0.23 \\
M & 2.01 & \pm 0.29 \\
P & 1.28 & \pm 0.15 \\
S & 1.49 & \pm 0.24 \\
T & 2.25 & \pm 0.37 \\
N & 2.36 & \pm 0.14 \\
Q & 2.50 & \pm 0.28 \\
D & 1.71 & \pm 0.25 \\
E & 2.02 & \pm 0.25 \\
H & 2.47 & \pm 0.39 \\
K & 2.06 & \pm 0.23 \\
R & 4.13 & \pm 0.20 \\
EV & 0.85 & \pm 0.12 \\
\hline
\end{tabular}

a): Values represent the initial rates of the ABTS oxidation reaction (mAU: milli-absorbance unit). Experimental details are given in chapter 1.6 . 
Table S3. Overview of nitrobindin variants discussed in this study.

\begin{tabular}{ll}
\hline Nitrobindin variant & Amino acid substitutions relative to NB-wt \\
\hline NB4 & M75L; H76L; Q96C; M148L; H158L \\
NB4-H & M75L; H76L; Q96C; M148L \\
NB4-HR & M75L; H76R; Q96C; M148L \\
NB-R & H76R \\
\hline
\end{tabular}


Table S4. Comparison of the kinetic parameters with natural peroxidases, reengineered proteins, and other ArMs.

\begin{tabular}{|c|c|c|c|c|}
\hline Substrate & Enzyme & $K_{\mathrm{m}}[\mu \mathrm{M}]$ & $k_{\text {cat }}\left(\mathrm{s}^{-1}\right)$ & $k_{\text {cat }} / K_{\mathrm{m}}\left[\mathbf{M}^{-1} \mathbf{s}^{-1}\right]$ \\
\hline \multirow[t]{13}{*}{ ABTS } & MnPPIX@NB4 & $100 \pm 43$ & $1.8 \pm 0.3$ & 18,000 \\
\hline & MnPPIX@NB4-H & $108 \pm 29$ & $4.9 \pm 0.5$ & 45,400 \\
\hline & MnPPIX@NB4-HR & $267 \pm 55$ & $14.1 \pm 1.4$ & 52,800 \\
\hline & ArM: $\alpha, \alpha-1,2-\mathrm{Fe}(\mathrm{DoCPP})-13 \mathrm{G} 10^{18}$ & 10,000 & $2.5 \pm 0.2$ & 253 \\
\hline & DyP: SlDtpA ${ }^{19}$ & $730 \pm 20$ & $1.12 \pm 0.2$ & 1,530 \\
\hline & DyP: $P p$ DyP $\mathrm{P}^{20}$ & $2,500 \pm 300$ & $23 \pm 2$ & 9,200 \\
\hline & DyP: $T v \mathrm{DyP} 1^{21}$ & $293 \pm 50$ & $582.1 \pm 0.1$ & $1,990,000$ \\
\hline & WT sperm whale myoglobin $(\mathrm{MyG})^{22}$ & $34 \pm 7$ & $2.6 \pm 0.1$ & 76,500 \\
\hline & $\mathrm{F} 43 \mathrm{Y} \mathrm{MyG}^{22}$ & $93 \pm 9$ & $29 \pm 1$ & 312,000 \\
\hline & $\mathrm{F} 43 \mathrm{Y} / \mathrm{T} 67 \mathrm{R} \mathrm{MyG}^{22}$ & $43 \pm 5$ & $44 \pm 2$ & $1,023,000$ \\
\hline & $\mathrm{F} 43 \mathrm{Y} / \mathrm{T} 67 \mathrm{R} / \mathrm{F} 138 \mathrm{~W} \mathrm{MyG}^{23}$ & $16 \pm 2$ & $31.5 \pm 0.6$ & $1,970,000$ \\
\hline & $\mathrm{HRP}^{\mathrm{a}) 24}$ & $3,800 \pm 700$ & $57.3 \pm 1.8$ & 15,180 \\
\hline & $\mathrm{HRP}^{\mathrm{b}) 25}$ & $800 \pm 10$ & $4,100 \pm 100$ & $5,125,000$ \\
\hline \multirow[t]{4}{*}{$\mathbf{S A}$} & MnPPIX@NB4 & $37 \pm 8$ & $0.25 \pm 0.02$ & 6,800 \\
\hline & MnPPIX@NB4-H & $21 \pm 2$ & $0.40 \pm 0.01$ & 19,000 \\
\hline & MnPPIX@NB4-HR & $104 \pm 17$ & $1.76 \pm 0.14$ & 16,900 \\
\hline & DyP: $P p \mathrm{Dy}^{26}$ & $48 \pm 8$ & 0.06 & 1,200 \\
\hline \multirow[t]{7}{*}{ DMP } & MnPPIX@NB4 & $13 \pm 3$ & $0.23 \pm 0.01$ & 17,700 \\
\hline & MnPPIX@NB4-H & $32 \pm 7$ & $0.91 \pm 0.06$ & 28,400 \\
\hline & MnPPIX@NB4-HR & $129 \pm 30$ & $2.30 \pm 0.27$ & 17,800 \\
\hline & ArM: Xln10A-Fe(TpCPP $)^{27}$ & 450 & 0,1 & 360 \\
\hline & DyP: SlDtpA ${ }^{19}$ & $5,600 \pm 100$ & $0.32 \pm 0.02$ & 57 \\
\hline & DyP: $P p \mathrm{Dy}^{20}$ & $70 \pm 20$ & $0.05 \pm 0.01$ & 700 \\
\hline & DyP: $T v \mathrm{DyP} 1^{21}$ & $1,026 \pm 79$ & $87.40 \pm 0.08$ & 85,200 \\
\hline
\end{tabular}

a) $\mathrm{pH}=7$;b) $\mathrm{pH}=4.6$. 


\section{Nucleotide and amino acid sequences}

\section{DNA sequence of the NB-wt open reading frame}

Codons of the amino acid positions 76 and 158 are highlighted.

ATGTGGAGCCACCCGCAGTTCGAAAAAAATCAACTGCAACAACTGCAAAATCCGGGCGAGAGTCC GCCGGTTCATCCGTTCGTGGCACCGCTGTCCTATCTGCTGGGTACCTGGCGCGGCCAGGGTGAAG GCGAGTATCCGACCATTCCGAGCTTTCGCTATGGCGAAGAGATCCGTTTCAGCCATTCGGGTAAA CCGGTGATTGCCTATACCCAAAAAACGTGGAAACTGGAATCGGGTGCACCGATGCACGCAGAGAG TGGTTATTTTCGCCCGCGTCCGGATGGTTCTATTGAAGTGGTTATCGCACAGTCGACCGGTCTGG TGGAAGTTCAAAAAGGCACGTATAATGTGGATGAGCAGAGTATTAAACTGAAATCTGACCTGGTG GGCAACGCGTCCAAAGTTAAAGAAATCAGCCGCGAATTCGAGCTGGTTGACGGTAAACTGAGTTA TGTGGTTCGTATGAGCACGACCACGAATCCGCTGCAACCGCACCTGAAAGCCATCCTGGACAAAC TGTAA

\section{Amino acid sequence of NB-wt}

The $N$-terminal leader peptide consisting of the Strep-tag II affinity tag is shown in lower case letters and was not considered in amino acid numbering. Position 76 and 158 are highlighted. The amino acid substitutions of various nitrobindin variants are summarized in Table S3.

mwshpqfekNQLQQLQNPGESPPVHPFVAPLSYLLGTWRGQGEGEYPTIPSFRYGEEIRFSHS GKPVIAYTQKTWKLESGAPMHAESGYFRPRPDGSIEVVIAQSTGLVEVQKGTYNVDEQSI KLKSDLVGNASKVKEISREFELVDGKLSYVVRMSTTTNPLQPHLKAILDKL

\section{DNA sequence of the SpyTag-functionalized sfGFP open reading frame}

AtgGTTCCTACTATTGTTATGgTGGACGCCTACAAACGCTATAAAGGATCAGAAAACCTGTATTT TCAGGGAGGTGGTTCAGGCCGTAAAGGCGAAGAGCTGTTCACTGGTGTCGTCCCTATTCTGGTGG AACTGGATGGTGATGTCAACGGTCATAAGTTTTCCGTGCGTGGCGAGGGTGAAGGTGACGCAACT AATGGTAAACTGACGCTGAAGTTCATCTGTACTACTGGTAAACTGCCGGTACCTTGGCCGACTCT GGTAACGACGCTGACTTATGGTGTTCAGTGCTTTGCTCGTTATCCGGACCATATGAAGCAGCATG ACTTCTTCAAGTCCGCCATGCCGGAAGGCTATGTGCAGGAACGCACGATTTCCTTTAAGGATGAC 
GGCACGTACAAAACGCGTGCGGAAGTGAAATTTGAAGGCGATACCCTGGTAAACCGCATTGAGCT GAAAGGCATTGACTTTAAAGAAGACGGCAATATCCTGGGCCATAAGCTGGAATACAATTTTAACA GCCACAATGTTTACATCACCGCCGATAAACAAAAAAATGGCATTAAAGCGAATTTTAAAATTCGC CACAACGTGGAGGATGGCAGCGTGCAGCTGGCTGATCACTACCAGCAAAACACTCCAATCGGTGA TGGTCCTGTTCTGCTGCCAGACAATCACTATCTGAGCACGCAAAGCGTTCTGTCTAAAGATCCGA ACGAGAAACGCGATCATATGGTTCTGCTGGAGTTCGTAACCGCAGCGGGCATCACGCATGGTATG GATGAACTGTACAAAGGCTCCGGCTCCGGCTCCCACCATCACCATCACCATTGA

\section{Amino acid sequence of SpyTag-functionalized sfGFP}

MVPTIVMVDAYKRYKGSENLYFQGGGSGRKGEELFTGVVPILVELDGDVNGHKFSVRG EGEGDATNGKLTLKFICTTGKLPVPWPTLVTTLTYGVQCFARYPDHMKQHDFFKSAMPE GYVQERTISFKDDGTYKTRAEVKFEGDTLVNRIELKGIDFKEDGNILGHKLEYNFNSHNV YITADKQKNGIKANFKIRHNVEDGSVQLADHYQQNTPIGDGPVLLPDNHYLSTQSVLSK DPNEKRDHMVLLEFVTAAGITHGMDELYKGSGSGSHHHHHH

\section{DNA sequence of the SpyCatcher open reading frame}

The codon of the cysteine for microgel conjugation is highlighted.

ATGTGCAGCGGCTGGAGCCACCCGCAGTTCGAAAAAAGCGGCGTAACCACCTTATCAGGTTTATC AGGTGAGCAAGGTCCGTCCGGTGATATGACAACTGAAGAAGATAGTGCTACCCATATTAAATTCT CAAAACGTGATGAGGACGGCCGTGAGTTAGCTGGTGCAACTATGGAGTTGCGTGATTCATCTGGT

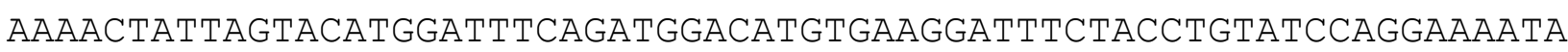
TACATTTGTCGAAACCGCAGCACCAGACGGTTATGAGGTAGCAACTGCTATTACCTTTACAGTTA AtGAgCAAgGTCAGgtTACTGTAAATGGCGAAgCAACTAAAgGTGACGCTCATACTTAA 


\section{Amino acid sequence of the SpyCatcher}

The cysteine residue used for microgel conjugation is highlighted. The $N$-terminal leader peptide containing of the cysteine residue is shown in lower case letters.

mcsgwshpqfeksgVTTLSGLSGEQGPSGDMTTEEDSATHIKFSKRDEDGRELAGATMELRDS SGKTISTWISDGHVKDFYLYPGKYTFVETAAPDGYEVATAITFTVNEQGQVTVNGEATK GDAHT

\section{DNA sequence of the NB4-H-SpyTag open reading frame}

ATGTGGAGCCACCCGCAGTTCGAAAAAAATCAACTGCAACAACTGCAAAATCCGGGCGAGAGTCC GCCGGTTCATCCGTTCGTGGCACCGCTGTCCTATCTGCTGGGTACCTGGCGCGGCCAGGGTGAAG GCGAGTATCCGACCATTCCGAGCTTTCGCTATGGCGAAGAGATCCGTTTCAGCCATTCGGGTAAA CCGGTGATTGCCTATACCCAAAAAACGTGGAAACTGGAATCGGGTGCACCGCTGCTGGCAGAGAG TGGTTATTTTCGCCCGCGTCCGGATGGTTCTATTGAAGTGGTTATCGCATGCTCGACCGGTCTGG TGGAAGTTCAAAAAGGCACGTATAATGTGGATGAGCAGAGTATTAAACTGAAATCTGACCTGGTG GGCAACGCGTCCAAAGTTAAAGAAATCAGCCGCGAATTCGAGCTGGTTGACGGTAAACTGAGTTA TGTGGTTCGTCTGAGCACGACCACGAATCCGCTGCAACCGCACCTGAAAGCCATCCTGGACAAAC TGGGTAGTGGTGAAAGTGGTGTGCCTACTATCGTGATGGTGGACGCCTACAAGCGTTACAAGGGC AGCTAA

\section{Amino acid sequence of NB4-H-SpyTag}

The $N$-terminal leader peptide consisting of the Strep-tag II affinity tag is shown in lower case letters.

mwshpqfekNQLQQLQNPGESPPVHPFVAPLSYLLGTWRGQGEGEYPTIPSFRYGEEIRFSHS GKPVIAYTQKTWKLESGAPLLAESGYFRPRPDGSIEVVIACSTGLVEVQKGTYNVDEQSI KLKSDLVGNASKVKEISREFELVDGKLSYVVRLSTTTNPLQPHLKAILDKLGSGESGVPTI VMVDAYKRYKGS 


\section{References}

1. Boucher, L. J. Manganese Porphyrin Complexes. I. Synthesis and Spectroscopy of Manganese(III) Protoporphyrin IX Dimethyl Ester Halides. J. Am. Chem. Soc. 1968, 90, 6640-6645.

2. Krieger, E.; Koraimann, G.; Vriend, G. Increasing the Precision of Comparative Models with YASARA NOVA - A Self-Parameterizing Force Field. Proteins: Struct., Funct., Bioinf. 2002, 47, 393-402.

3. Canutescu, A. A.; Shelenkov, A. A.; Dunbrack Jr, R. L. A Graph-Theory Algorithm for Rapid Protein Side-Chain Prediction. Protein Sci. 2003, 12, 2001-2014.

4. Bianchetti, C. M.; Blouin, G. C.; Bitto, E.; Olson, J. S.; Phillips, G. N. The Structure and NO Binding Properties of the Nitrophorin-like Heme-Binding Protein from Arabidopsis thaliana Gene Locus At1g79260.1. Proteins: Struct., Funct., Bioinf. 2010, 78, 917-931.

5. Miyamoto, S.; Kollman, P. A. Settle: An Analytical Version of the SHAKE and RATTLE Algorithm for Rigid Water Models. J. Comput. Chem. 1992, 13, 952-962.

6. Maier, J. A.; Martinez, C.; Kasavajhala, K.; Wickstrom, L.; Hauser, K. E.; Simmerling, C. ff14SB: Improving the Accuracy of Protein Side Chain and Backbone Parameters from ff99SB. J. Chem. Theory Comput. 2015, 11, 3696-3713.

7. Wang, J.; Wolf, R. M.; Caldwell, J. W.; Kollman, P. A.; Case, D. A. Development and Testing of a General Amber Force Field. J. Comput. Chem. 2004, 25, 1157-1174.

8. Jakalian, A.; Jack, D. B.; Bayly, C. I. Fast, Efficient Generation of High-Quality Atomic Charges. AM1-BCC Model: II. Parameterization and Validation. J. Comput. Chem. 2002, $23,1623-1641$.

9. Krieger, E.; Darden, T.; Nabuurs, S. B.; Finkelstein, A.; Vriend, G. Making Optimal Use of Empirical Energy Functions: Force-Field Parameterization in Crystal Space. Proteins: Struct., Funct., Bioinf. 2004, 57, 678-683.

10. Abraham, M. J.; Murtola, T.; Schulz, R.; Páll, S.; Smith, J. C.; Hess, B.; Lindahl, E. GROMACS: High Performance Molecular Simulations Through Multi-Level Parallelism from Laptops to Supercomputers. SoftwareX 2015, 1, 19-25.

11. Fukumoto, K.; Onoda, A.; Mizohata, E.; Bocola, M.; Inoue, T.; Schwaneberg, U.; Hayashi, T. Rhodium-Complex-Linked Hybrid Biocatalyst: Stereo-Controlled Phenylacetylene Polymerization within an Engineered Protein Cavity. ChemCatChem 2014, 6, 1229-1235. 
12. Kille, S.; Acevedo-Rocha, C. G.; Parra, L. P.; Zhang, Z.-G.; Opperman, D. J.; Reetz, M. T.; Acevedo, J. P. Reducing Codon Redundancy and Screening Effort of Combinatorial Protein Libraries Created by Saturation Mutagenesis. ACS Synth. Biol. 2013, 2, 83-92.

13. Gibson, D. G.; Young, L.; Chuang, R.-Y.; Venter, J. C.; Hutchison, C. A.; Smith, H. O. Enzymatic Assembly of DNA Molecules up to Several Hundred Kilobases. Nat. Methods 2009, 6, 343-345.

14. Key, H. M.; Dydio, P.; Clark, D. S.; Hartwig, J. F. Abiological Catalysis by Artificial Haem Proteins Containing Noble Metals in Place of Iron. Nature 2016, 534, 534-537.

15. Vu, N. T.; Moriwaki, Y.; Caaveiro, J. M. M.; Terada, T.; Tsutsumi, H.; Hamachi, I.; Shimizu, K.; Tsumoto, K. Selective Binding of Antimicrobial Porphyrins to the Heme-Receptor IsdHNEAT3 of Staphylococcus aureus. Protein Sci. 2013, 22, 942-953.

16. Gau, E.; Mate, D. M.; Zou, Z.; Oppermann, A.; Töpel, A.; Jakob, F.; Wöll, D.; Schwaneberg, U.; Pich, A. Sortase-Mediated Surface Functionalization of Stimuli-Responsive Microgels. Biomacromolecules 2017, 18, 2789-2798.

17. Keeble, A. H.; Banerjee, A.; Ferla, M. P.; Reddington, S. C.; Anuar, I. N. A. K.; Howarth, M. Evolving Accelerated Amidation by SpyTag/SpyCatcher to Analyze Membrane Dynamics. Angew. Chem. Int. Ed. 2017, 56, 16521-16525.

18. de Lauzon, S.; Mansuy, D.; Mahy, J.-P. Coordination Chemistry of Oron(III)-PorphyrinAntibody Complexes. Eur. J. Biochem. 2002, 269, 470-480.

19. Chaplin, A. K.; Wilson, M. T.; Worrall, J. A. R. Kinetic Characterisation of a Dye Decolourising Peroxidase from Streptomyces lividans: New Insight into the Mechanism of Anthraquinone Dye Decolourisation. Dalton Trans. 2017, 46, 9420-9429.

20. Brissos, V.; Tavares, D.; Sousa, A. C.; Robalo, M. P.; Martins, L. O. Engineering a Bacterial DyP-Type Peroxidase for Enhanced Oxidation of Lignin-Related Phenolics at Alkaline pH. ACS Catal. 2017, 7, 3454-3465.

21. Amara, S.; Perrot, T.; Navarro, D.; Deroy, A.; Benkhelfallah, A.; Chalak, A.; Daou, M.; Chevret, D.; Faulds, C. B.; Berrin, J.-G.; Morel-Rouhier, M.; Gelhaye, E.; Record, E. Enzyme Activities of Two Recombinant Heme-Containing Peroxidases, $T v$ DyP1 and $T v \mathrm{VP} 2$, Identified from the Secretome of Trametes versicolor. Appl. Environ. Microbiol. 2018, 84, e02826-17.

22. Liu, C.; Yuan, H.; Liao, F.; Wei, C.-W.; Du, K.-J.; Gao, S.-Q.; Tan, X.; Lin, Y.-W. Unique Tyr-Heme Double Cross-Links in F43Y/T67R Myoglobin: An Artificial Enzyme with a 
Peroxidase Activity Comparable to that of Native Peroxidases. Chem. Commun. 2019, 55, 6610-6613.

23. Liao, F.; Xu, J.-K.; Luo, J.; Gao, S.-Q.; Wang, X.-J.; Lin, Y.-W. Bioinspired Design of an Artificial Peroxidase: Introducing Key Residues of Native Peroxidases into F43Y Myoglobin with a Tyr-Heme Cross-Link. Dalton Trans. 2020, 49, 5029-5033.

24. Rodriguez-Lopez, J. N.; Smith, A. T.; Thorneley, R. N. F. Role of Arginine 38 in Horseradish Peroxidase: A Critical Residue for Substrate Binding and Catalysis. J. Biol. Chem. 1996, 271, 4023-4030.

25. Savenkova, M. I.; Kuo, J. M.; Ortiz de Montellano, P. R. Improvement of Peroxygenase Activity by Relocation of a Catalytic Histidine within the Active Site of Horseradish Peroxidase. Biochemistry 1998, 37, 10828-10836.

26. Santos, A.; Mendes, S.; Brissos, V.; Martins, L. O. New Dye-Decolorizing Peroxidases from Bacillus subtilis and Pseudomonas putida MET94: Towards Biotechnological Applications. Appl. Microbiol. Biotechnol. 2014, 98, 2053-2065.

27. Ricoux, R.; Dubuc, R.; Dupont, C.; Marechal, J.-D.; Martin, A.; Sellier, M.; Mahy, J.-P. Hemozymes Peroxidase Activity of Artificial Hemoproteins Constructed from the Streptomyces lividans Xylanase A and Iron(III)-Carboxy-Substituted Porphyrins. Bioconjugate Chem. 2008, 19, 899-910. 